\title{
Transition operators
}

\section{on co-compact $G$-spaces}

\section{Laurent Saloff-Coste and Wolfgang Woess}

\section{Dedicated to the memory of Bob Brooks}

\begin{abstract}
We develop methods for studying transition operators on metric spaces that are invariant under a co-compact group which acts properly. A basic requirement is a decomposition of such operators with respect to the group orbits. We then introduce "reduced" transition operators on the compact factor space whose norms and spectral radii are upper bounds for the $L^{p}$-norms and spectral radii of the original operator. If the group is amenable then the spectral radii of the original and reduced operators coincide, and under additional hypotheses, this is also sufficient for amenability. Further bounds involve the modular function of the group.

In this framework, we prove among other things that the bottom of the spectrum of the Laplacian on a co-compact Riemannian manifold is 0 if and only if the group is amenable and unimodular. The same result holds for Euclidean simplicial complexes. On a geodesic, proper metric space with co-compact isometry group action, the averaging operator over balls with a fixed radius has norm equal to 1 if and only if the group is amenable and unimodular. The technique also allows explicit computation of spectral radii when the group is amenable.
\end{abstract}

\section{Introduction: Four theorems in search of a common theory}

We start by stating four theorems, not in chronological order, concerning Laplace operators on graphs and manifolds. The purpose of this paper is to develop a common theory into which these results fit naturally.

2000 Mathematics Subject Classification: 22F50, 43A07, 47A30, 58C40, 58J05.

Keywords: $G$-space, proper action, isometries, invariant transition operator, factor space, amenability, unimodularity, Riemannian manifold, Laplacian, Euclidean simplicial complex, geodesic metric space. 
Theorem I (Soardi and Woess [38]). Let $X$ be a vertex-transitive, connected, locally finite graph and $P$ the simple random walk on $X$. Then the spectral radius $\rho(P)=1$ if and only if some (equivalently, every) closed, transitive group of automorphisms of $X$ is both amenable and unimodular.

Here, automorphisms are self-isometries of $X$ with respect to the discrete graph metric. "Closed" refers to the topology of point-wise convergence, under which the automorphism group is locally compact and totally disconnected. The simple random walk on any locally finite, connected graph $X$ is the Markov chain with transition operator on $X$ given by $p(x, y)=1 / \operatorname{deg}(x)$, if $y$ is a neighbour of $x$, and $p(x, y)=0$, otherwise. The degree $\operatorname{deg}(x)$ is the number of neighbours of $x$, and $\rho(P)=\lim _{n} p^{(2 n)}(x, x)^{1 / 2 n}$ is the spectral radius of $P$ acting on $\ell^{2}(X, d e g)$.

For Riemannian manifolds, there is the following analogue.

Theorem II (Pittet [28]). Let $\mathcal{M}$ be a connected non-compact Riemannian manifold which is homogeneous in the sense that its group of isometries $G$ acts transitively. Then 0 is in the $L^{2}$-spectrum of the Laplacian of $\mathcal{M}$ if and only if $G$ is amenable and unimodular.

By the Laplacian of $\mathcal{M}$ we mean the Laplace-Beltrami operator acting on functions. Pittet's paper concerns the decay of the heat kernel, and it contains more than this theorem; it is likely that the latter was known to the specialists before, but we are not aware of an earlier explicit reference.

In general, we equip the full isometry group of a metric space $X$ with the topology of uniform convergence on compact sets. We say that a group of isometries $G$ of $X$ is quasi-transitive, or co-compact, if it acts with compact factor space $G \backslash X$. A metric space is called quasi-transitive, or co-compact, if such a group exists. In case of a graph, this means that $G$ acts with finitely many orbits on the vertex set.

Theorem III (Salvatori [35]). Let $X$ be a quasi-transitive, connected, locally finite graph and $P$ the simple random walk on $X$. Then $\rho(P)=1$ if and only if some (equivalently, every) closed, quasi-transitive group of automorphisms of $X$ is both amenable and unimodular.

Finally, the following is the earliest of the four theorems that we are referring to.

Theorem IV (Brooks [5]). Let $\mathcal{M}$ be the universal cover of the compact Riemannian manifold $\mathcal{M}_{0}$. Then 0 is in the $L^{2}$-spectrum of the Laplacian of $\mathcal{M}$ if and only if the fundamental group of $\mathcal{M}_{0}$ is amenable.

When comparing Theorems II and IV, recall that the fundamental group of a compact manifold is of course discrete, hence unimodular. Brooks' the- 
orem was extended to Schrödinger operators on normal coverings of compact manifolds by Kobayashi, Ono and Sunada [24], and the analogue for Schrödinger operators on coverings of finite graphs was proved by Sy and Sunada [40]. For graph Laplacians, the latter result follows from Salvatori's Theorem III.

The reader will note that Theorem IV is not the complete generalization of Theorem II to the quasi-transitive (三 co-compact) case that one would expect in analogy with Theorems I and III treating graphs. Here we shall complete the picture: two among our main results are the following.

(8.1) Theorem. Let $\mathcal{M}$ be a complete, co-compact Riemannian manifold. Then 0 is in the $L^{2}$-spectrum of the Laplacian of $\mathcal{M}$ if and only if some (equivalently, every) closed, quasi-transitive group of isometries of $\mathcal{M}$ is both amenable and unimodular.

In Theorem 8.1, the groups in consideration are of course Lie groups, possibly 0-dimensional, i.e., finitely generated discrete. Theorem IV of Brooks [5] arises as a special case. It is noteworthy that our proof of Theorem 8.1 uses almost no specific facts from Riemannian geometry besides a basic long distance estimate for the heat kernel. It follows that Theorem 8.1 extends beyond the Riemannian setting in several directions. In particular, we treat Euclidean simplicial complexes; see (8.4) for a precise description of the setup.

(8.5) Theorem. Let $X$ be a co-compact connected $d$-dimensional simplicial complex. Then 0 is in the $L^{2}$-spectrum of the Laplacian of $X$ if and only if some (equivalently, every) closed, quasi-transitive group of isometries of $X$ is both amenable and unimodular.

A simple but quite general example of interest which illustrates the problems studied in this paper and that the reader may keep in mind is the following. Let $X$ be a locally compact metric space on which a group $G$ acts by isometries and with compact quotient. Consider the "averaging" operator $f \mapsto K f$ where $K f(x)$ is the mean of $f$ over the ball of radius 1 around $x$. By analogy to the results described above, one would like to characterize when the $\left(L^{p}\right)$ spectral radius of such an operator is strictly less than 1 and, in some specific instance, compute explicitly that spectral radius. The techniques and results of this paper apply indeed to that type of situation: See Examples 3.6 (averaging operator in the hyperbolic plane), 8.7 (averaging operator on a simplicial homogeneous tree), and Theorem 8.6.

The setting that we shall use is that of transition operators. We start with a proper metric space $(X, d)$, where proper means that closed balls in $X$ are compact. 
(1.1) Definition. A transition operator on $X$ is an operator of the form

$$
K f(x)=\int_{X} f(y) K(x, d y)
$$

acting on non-negative measurable functions $f: X \rightarrow \mathbb{R}$, where $K(x,$.$) ,$ $x \in X$, is a family of non-negative Radon measures on $X$ such that the mapping $x \mapsto K(x, B)$ is measurable for every $B \in \mathcal{B}(X)$, the collection of Borel subsets of $X$.

In general, $K f$ is defined for all measurable functions $f$ for which the above integral exists. We always assume that $K$ is non-degenerate, that is, $K(x, X)>0$ for all $x$. All measures appearing in this paper are assumed to be Radon measures. (Since every $\sigma$-finite measure on $X$ is a Radon measure, see e.g. Rudin [31, Thm. 2.18], this is a natural assumption.)

The other basic object is a locally compact group $G$ that acts properly on $X$ by homeomorphisms with compact factor space $I=G \backslash X$. Recall that a proper $G$-action is one where the set $\{g \in G: g A \cap B \neq \emptyset\}$ is compact in $G$ for all compact sets $A, B \subset X$; see e.g. tom Dieck [15] for equivalent formulations. Note that these assumptions are satisfied when $X$ is a proper metric space and $G$ a closed subgroup of the isometry group of $X$, equipped with the topology of uniform convergence on compact sets.

The fundamental assumption is G-invariance of the transition operator, that is,

$$
K(g x, g B)=K(x, B) \quad \text { for all } g \in G, x \in X, B \in \mathcal{B}(X) \text {. }
$$

There are natural choices of Radon measures supported on the whole of $X$ which are invariant under the action of $G$ (only in the special case when $G$ acts transitively on $X$, the invariant measure is unique up to normalization). We shall (carefully) choose one of these measures and denote it by $d_{X}$.

We are interested in the norms $\sigma_{p}(K)=\sigma_{p}\left(K, d_{X}\right)=\|K\|_{p \rightarrow p}$ and spectral radii $\rho_{p}(K)=\rho_{p}\left(K, d_{X}\right)$ of $K$ acting on the spaces $L^{p}\left(X, d_{X}\right)$, $1<p<\infty$, and we want to relate these numbers to properties of the group $G$ (amenability, unimodularity). Since $|K f| \leq K|f|$, for norms and spectral radii it is sufficient to consider the action of $K$ on the positive cones $L_{+}^{p}\left(X, d_{X}\right)$, and in this sense, those numbers are always well-defined in $[0, \infty]$.

The "historical" root of this paper is the Theorem of Kesten [23] and Day [12], saying that for a symmetric random walk on a finitely generated, discrete group, the spectral radius is equal to 1 if and only if the group is amenable. This was extended to convolution operators on locally compact groups by Berg and Christensen [1], [2] and by Derriennic and Guivarc'h [14]. 
Another result related to the problems treated in this paper is Carl Herz' "principe de majoration" [20] and the well known transfer result that can be informally stated as follows: If $G$ is a locally compact group, $H$ is a closed amenable subgroup of $G$ and $\mu$ is a positive measure on $G$, then the $L^{p}$ norm of the operator defined by convolution with $\mu$ is equal to the $L^{p}$-norm of the induced operator on $G / H$. See Herz [21], Lohoué [25], Guivarc'h [19], and Cowling [10]. Note, however, the difference between a $G$-invariant transition operator, as considered in the present paper, and a left convolution operator on a $G$-space. The latter is in general not $G$-invariant, while this is the case for typical operators on a $G$-space, such as the ones considered in Theorems 8.1, 8.5 and 8.6.

The methods that we use in the present paper are based on Saloff-Coste and Woess [34], see also Saloff-Coste and Woess [33]. In those two papers, we refined the results of Soardi and Woess [38] and Salvatori [35] in the setting of transition operators (random walks) on graphs. The general setting that we study here, where both the group and the $G$-space are allowed to be nondiscrete, presents various additional obstacles that we are going to tackle here.

Here is an outline of this paper.

In $\S 2$, we consider a $G$-invariant transition operator $K$ between two possibly different, but homogeneous $G$-spaces $X$ and $Y$ (i.e., $G$ acts properly and transitively on both of them). Considering $K$ as an operator from $L^{p}\left(Y, d_{Y}\right)$ to $L^{p}\left(X, d_{X}\right)$, we extend a basic idea of Soardi and Woess [38] and lift $K$ to an operator $\mathcal{K}$ on $L^{p}\left(G, d_{G}\right)$. This $\mathcal{K}$ turns out to be a right convolution operator on $G$, and computing its norm with respect to the left Haar measure $d_{G}$ involves the modular function of $G$. We then apply results of Reiter, see Reiter and Stegemann [30], and Berg and Christensen [1], [2] that relate the norms of convolution operators with amenability. This leads to two upper bounds on the $L^{p}$-norm of $\left.K, \sigma_{p}\left(K, d_{X}, d_{Y}\right)\right) \leq a_{p}(K) \leq b(K)^{1 / q} b\left(K^{*}\right)^{1 / p}$, with explicit expressions for the numbers $a_{p}(K)$ and the total masses $b(K)$ and $b\left(K^{*}\right)$ of $K$ and its adjoint $K^{*}$. (Here, $\frac{1}{p}+\frac{1}{q}=1$.) Furthermore, we obtain conditions when equality holds that involve amenability (Theorem 2.12) and unimodularity (Proposition 2.17), respectively.

In $\S 3$, we consider the specific situation where we have a $G$-invariant transition operator on a single homogeneous $G$-space. Besides norms, we can now also include spectral radii in our considerations. The main results are subsumed in Theorem 3.2. Theorem II of Pittet [28] arises as an immediate corollary. When $G$ is amenable, Theorem 3.2 also yields an explicit formula for the norm and the spectral radius of $K$ acting on $L^{p}\left(X, d_{X}\right)$ (the two coincide!), and at the end of $\S 3$, we illustrate this by a concrete computational example. 
In $\S \S 4-8$ we then develop a general theory for transition operators in the co-compact situation.

Two basic measure-theoretic requirements are outlined in $\S 4$. The first is a construction, resp. decomposition, of a $G$-invariant measure $d_{X}$ on the co-compact space $X$ of the form $d_{X}=\int_{I} d_{X_{i}} d \lambda(i)$, where $\lambda$ is a measure on the factor space $I$, the $X_{i}(i \in I)$ are the $G$-orbits, and $d_{X_{i}}$ is the suitably (!) normalized $G$-invariant measure on $X_{i}$. The second, more complicated question concerns the existence of an analogous decomposition $K(x, \cdot)=$ $\int_{I} K_{j}(x, \cdot) d \lambda(j)$, where each $K_{j}$ is a $G$-invariant transition operator from $X$ to $X_{j}$. The respective details and proofs are deferred to the Appendix ( $(9)$.

In $\S 5$, we start with such a decomposition of $K$ and write $K_{i, j}$ for the restriction of $K_{j}(x, \cdot)$ to $x \in X_{i}$. The results of $\S 2$ can then be applied to the family of transition operators $K_{i, j}, i, j \in I$. The numbers $a_{p}\left(K_{i, j}\right), b\left(K_{i, j}\right)$ and $b\left(K_{i, j}^{*}\right)$ define the kernels, with respect to the measure $\lambda$, of transition operators $\mathfrak{A}_{p}[K], \mathfrak{B}[K]$ and $\mathfrak{B}_{*}[K]$ (respectively) on $I$, and we find that their $L^{p}$-spectral radii satisfy

$$
\rho_{p}(K) \leq \rho_{p}\left(\mathfrak{A}_{p}[K]\right) \leq \rho_{p}(\mathfrak{B}[K])^{1 / q} \rho_{q}\left(\mathfrak{B}_{*}[K]\right)^{1 / p},
$$

and the same holds for the respective norms. See Theorem 5.3 and Proposition 5.5, where we also show that the first, resp. second, inequality for the spectral radii is an equality when $G$ is amenable, resp. unimodular. (The first is also true for norms).

In $\S 6$, we ask under which circumstances the reversed implications are also true, i.e., that equality in the first or second of the above inequalities implies amenability, resp. unimodularity of $G$, or at least of a suitable subgroup of $G$. Results of this type can be obtained if the "reduced" operators $\mathfrak{A}_{p}[K], \mathfrak{B}[K]$ and $\mathfrak{B}_{*}[K]$ are compact, $\lambda$-irreducible operators on $L^{p}(I, \lambda)$. The main results are Theorem 6.7 and Corollary 6.9 regarding amenability, and Proposition 6.10 regarding unimodularity.

$\S 7$ is devoted to the most practicable situation, namely, when $K(x, \cdot)$ has a density $k(x, y)$ with respect to $d_{X}$, and $G$ acts by isometries. If the function $k(\cdot, \cdot)$ is bounded on compact subsets of $X \times X$ and both $K$ and $K^{*}$ have bounded tails, then $\mathfrak{B}[K], \mathfrak{B}_{*}[K]$ and $\mathfrak{A}_{p}[K]$ have bounded densities. Therefore they are compact operators. Thus, we obtain a rather general set of natural geometric and analytic conditions under which the results of $\S \S 5-6$ apply (Theorem 7.10).

In $\S 8$, we exhibit several applications of the preceding results. Theorems 8.1 and 8.5 on the Laplacian of a co-compact Riemannian manifold, resp. simplicial complex, arise as corollaries of Theorem 7.10: the density of the transition operator which we use here is the heat kernel at time $t=1$, which is continuous and has uniform Gaussian decay. 
In the more general context of co-compact geodesic metric spaces, we show that for a fixed radius $r$, the averaging operator over $r$-balls has spectral radius equal to one if and only if the group is amenable and unimodular (Theorem 8.6). This transition operator corresponds to a random walk, where at each step, the walker - given that his actual position is $x$ - moves to a random point that is chosen according to $d_{X}$-equidistribution in the ball $B(x, r)$. This works more generally for co-compact $\delta$-geodesic metric spaces (see $\S 7$ for the definition), when $r>\delta$.

Already without the additional assumptions (compactness, irreducibility, density) used in $\S \S 6-7$, Theorem 5.3 yields that $\rho_{p}(K)=\rho_{p}\left(\mathfrak{A}_{p}[K]\right)$ and $\sigma_{p}(K)=\sigma_{p}\left(\mathfrak{A}_{p}[K]\right)$ when $G$ is amenable. Thus, computations reduce to those of norms and spectral radii of operators on the compact space $I$. This can be used, rather for spectral radii than for norms in general, to perform explicit computations in suitable cases. Since computations are space-consuming, only one - basic and typical - example of this type is considered in the final part of $\S 8$. We plan to present further examples and applications in forthcoming work.

The appendix ( $\S 9)$ completes $\S 4$ : it contains the measure-theoretic details concerning decompositions of the invariant measure and the transition operator over the group's orbits.

We would like to stress that our intention is to provide a mathematical framework into which the four introductory theorems as well as their extensions (manifolds, complexes, geodesic spaces) fit nicely. Thus, in the present paper, our aim is not merely to find the most rapid way towards the proofs of the theorems in $\S 8$, but also to provide a sufficiently complete picture of the framework. On the other hand, it is clear that some parts of this theory can be pushed towards further generality in various ways. We have tried to maintain a reasonable equilibrium in this respect.

\section{Transition operators between homogeneous spaces}

We shall first consider a slightly different general setup where $G$ acts transitively on two metric spaces $X, Y$. Again, $G$ is assumed locally compact and both actions have to be proper. We fix -once for all- a left Haar measure $d_{G}$ on $G$, and choose some $G$-invariant measures $d_{X}$ on $X$ and $d_{Y}$ on $Y$. Their existence and uniqueness up to normalization is guaranteed by Bourbaki [3, Chap. VII, $\S 7$, Cor. 2], see also Raghunathan [29, pp. 16-19].

Denote by $G_{x}$ the stabilizer of $x$ in $G$. By properness of the action, this is a compact subgroup of $G$, and it carries a (left and right) Haar measure with finite total mass that will be denoted $d_{G_{x}}$. Now let $\bar{x} \in X$, and for each $x \in X$, choose an element $g_{\bar{x}, x}$ with $g_{\bar{x}, x} \bar{x}=x$. If $F \in L^{1}(G, d g)$ then 
$x \mapsto \int_{G_{\bar{x}}} F\left(g_{\bar{x}, x} h\right) d_{G_{\bar{x}}} h$ defines a function in $L^{1}\left(X, d_{X}\right)$ that is independent of the particular choice of $g_{\bar{x}, x} \in G$ mapping $\bar{x}$ to $x$. We have the fundamental formula

$$
\int_{G} F(g) d_{G} g=\int_{X}\left(\int_{G_{\bar{x}}} F\left(g_{\bar{x}, x} h\right) d_{G_{\bar{x}}} h\right) d_{X} x
$$

see e.g. Bourbaki [3, Chap. VII, §2, Prop. 5] ${ }^{1}$ or Raghunathan [29, pp. 16-19]. In (2.1), the normalization of two of the three measures $d_{G}, d_{X}$ and $d_{G_{\bar{x}}}$ can be chosen freely, and this forces the third one. We shall always start with $d_{G}$ and $d_{X}$, thus forcing the normalization of $d_{G_{\bar{x}}}$. With this normalization, we set

$$
\left|G_{\bar{x}}\right|=\int_{G_{\bar{x}}} d_{G_{\bar{x}}} .
$$

The same applies to the action of $G$ on $Y$, replacing $g_{\bar{x}, x}$ by $g_{\bar{y}, y}$, where $\bar{y}, y \in Y$, and writing $G_{\bar{y}}$ for the stabilizer of $\bar{y} \in Y$. Observe however that $\left|G_{\bar{x}}\right|$ depends not only on $G_{\bar{x}}$, but also on the orbit to which $\bar{x}$ belongs. I.e., it may happen that $G_{\bar{x}}=G_{x^{\prime}}$ as subgroups of $G$ for different $\bar{x}, x^{\prime} \in X$, while (2.1) forces normalizations of the respective Haar measures such that $\left|G_{\bar{x}}\right| \neq\left|G_{x^{\prime}}\right|$. Thus, the notation $\left|G_{\bar{x}}\right|$ should more correctly be $\left|G_{\bar{x}}\right|_{\bar{x}}$, but we will omit this. See Examples 3.6 and 8.7 for situations of this type.

In the setting of two homogeneous $G$-spaces $X, Y$, we now consider a transition operator from $X$ to $Y$, i.e., a family $K(x, \cdot), x \in X$, of nonnegative Radon measures on $Y$ such that $K(\cdot, B)$ is measurable for every $B \in \mathcal{B}(Y)$. We assume $G$-invariance:

$$
K(g x, g B)=K(x, B) \quad \text { for all } g \in G, x \in X, B \in \mathcal{B}(Y),
$$

If $f \in \mathcal{C}_{00}(Y)$, the space of real-valued continuous functions on $Y$ with compact support, then

$$
K f(x)=\int_{Y} f(y) K(x, d y)
$$

defines a measurable function $X \rightarrow \mathbb{R}$. Our "minimal" assumption is that $K f$ is locally integrable for every $f \in \mathcal{C}_{00}(Y)$, i.e., $\int_{U} K f(x) d_{X} x$ exists and is finite for every compact $U \subset X$. We then call $K$ locally finite. (Typically, $K$ will be finite, i.e., the measure $K(x, \cdot)$ is finite for every $x \in X$.) Supposing that $K$ extends to a bounded linear operator from $L^{p}\left(Y, d_{Y}\right)$ to $L^{p}\left(X, d_{X}\right)$, we are interested in the corresponding norm

$$
\sigma_{p}(K)=\sigma_{p}\left(K, d_{X}, d_{Y}\right) \stackrel{\text { def }}{=}\|K\|_{p \rightarrow p}, \quad 1<p<\infty .
$$

\footnotetext{
${ }^{1}$ Use $X \leftrightarrow G, H \leftrightarrow G_{\bar{x}}, X / H \leftrightarrow X$ for the correspondence Bourbaki's $\leftrightarrow$ our setting
} 
As pointed out in the introduction, since $|K f| \leq K|f|$, the norm is always well defined in $[0, \infty]$ in terms of the action of $K$ on non-negative functions, even when $K$ is not $L^{p}$-bounded. Throughout this paper, we shall write $q$ for the exponent that satisfies $\frac{1}{p}+\frac{1}{q}=1$.

We now choose reference points $\bar{x} \in X$ and $\bar{y} \in Y$. Given $\bar{x}$, we define two operators $\mathcal{S}_{\bar{x}}: L^{p}\left(G, d_{G}\right) \rightarrow L^{p}\left(X, d_{X}\right)$ and $\mathcal{T}_{\bar{x}}: L^{p}\left(X, d_{X}\right) \rightarrow L^{p}\left(G, d_{G}\right)$ (for any $p \in[1, \infty]$ ) by

$$
\mathcal{S}_{\bar{x}} F(x)=\frac{1}{\left|G_{\bar{x}}\right|} \int_{G_{\bar{x}}} F\left(g_{\bar{x}, x} h\right) d_{G_{\bar{x}}} h \quad \text { and } \quad \mathcal{T}_{\bar{x}} f(g)=f(g \bar{x}) .
$$

Then we have the following.

(2.4) Lemma. (a) $\mathcal{S}_{\bar{x}} \mathcal{T}_{\bar{x}}$ is the identity operator.

$$
\left\|\mathcal{S}_{\bar{x}}\right\|_{p \rightarrow p}=\left|G_{\bar{x}}\right|^{-1 / p} \quad \text { and } \quad\left\|\mathcal{T}_{\bar{x}}\right\|_{p \rightarrow p}=\left|G_{\bar{x}}\right|^{1 / p} .
$$

(c) For all $f \in L^{p}\left(X, d_{X}\right), F \in L^{p}\left(G, d_{G}\right)$ and $g \in G$,

$$
\delta_{g} *\left(\mathcal{S}_{\bar{x}} F\right)=\mathcal{S}_{\bar{x}}\left(\delta_{g} * F\right) \quad \text { and } \quad \delta_{g} *\left(\mathcal{T}_{\bar{x}} f\right)=\mathcal{S}_{\bar{x}}\left(\delta_{g} * f\right)
$$

The proof is an easy exercise. Recall that $\delta_{g} * F\left(g^{\prime}\right)=F\left(g^{-1} g^{\prime}\right)$ and $\delta_{g} * f(x)=f\left(g^{-1} x\right)$. Furthermore, the operators $\mathcal{S}_{\bar{x}}$ and $\mathcal{T}_{\bar{x}}$ map continuous functions to continuous functions.

Before proceeding, we need some considerations regarding the modular function $\Delta$ of $G$, which plays a crucial role in this paper. Recall that this is an exponential, i.e., a continuous homomorphism from $G$ to $\left(\mathbb{R}^{+}, \cdot\right)$ with

$$
\Delta(\bar{g}) \int_{G} F(g \bar{g}) d_{G} g=\int_{G} F(g) d_{G} g=\int_{G} F\left(g^{-1}\right) \Delta\left(g^{-1}\right) d_{G} g
$$

for any $F \in L^{1}\left(G, d_{G}\right)$. For the following lemma, it is crucial that the normalization of the Haar measure on point stabilizers is as described after (2.1) above.

(2.6) Lemma. (a) If $f \in L^{1}\left(X, d_{X}\right)$ then for any $\bar{x} \in X$

$$
\int_{G} f(g \bar{x}) d_{G} g=\left|G_{\bar{x}}\right| \int_{X} f(x) d_{X} x .
$$

(b) For every $\bar{g} \in X$ and $\bar{x} \in X, \Delta(\bar{g})=\left|G_{\bar{x}}\right| /\left|G_{\bar{g} \bar{x}}\right|$.

(c) For every $\bar{g} \in G$ and $\bar{x} \in X$, if $F: G_{\bar{g} \bar{x}} \rightarrow \mathbb{R}$ is $d_{G_{\bar{g} \bar{x}}}$-integrable then

$$
\int_{G_{\bar{x}}} F\left(\bar{g} h \bar{g}^{-1}\right) d_{G_{\bar{x}}} h=\Delta(\bar{g}) \int_{G_{\bar{g} \bar{x}}} F(h) d_{G_{\bar{g} \bar{x}}} h .
$$


Proof. Using (2.1),

$$
\int_{G} f(g \bar{x}) d_{G} g=\int_{X} \int_{G_{\bar{x}}} f\left(g_{\bar{x}, x} h \bar{x}\right) d_{G_{\bar{x}}} h d_{X} x=\int_{X} f(x)\left|G_{\bar{x}}\right| d_{X} x .
$$

This proves (a). To see (b), we use (2.5) and a function $f$ satisfying $\int_{X} f(x) d_{X} x=1$ and get

$$
\left|G_{\bar{g} \bar{x}}\right|=\int_{G} f(g \bar{g} \bar{x}) d_{G} g=\Delta(\bar{g})^{-1} \int_{G} f(g \bar{x}) d_{G} g=\Delta(\bar{g})^{-1}\left|G_{\bar{x}}\right| .
$$

For proving (c), define a measure $\mu_{\bar{g} \bar{x}}$ on $G_{\bar{g} \bar{x}}$ by

$$
\int_{G_{\bar{g} \bar{x}}} F d \mu_{\bar{g} \bar{x}}=\int_{G_{\bar{x}}} F\left(\bar{g} h \bar{g}^{-1}\right) d_{G_{\bar{x}}} h .
$$

Let $\bar{h} \in G_{\bar{g} \bar{x}}$. Then

$$
\int_{G_{\bar{g} \bar{x}}} \delta_{\bar{h}} * F d \mu_{\bar{g} \bar{x}}=\int_{G_{\bar{x}}} F\left(\bar{g}\left(\bar{g}^{-1} \bar{h} \bar{g}\right) h \bar{g}^{-1}\right) d_{G_{\bar{x}}} h=\int_{G_{\bar{g} \bar{x}}} F d \mu_{\bar{g} \bar{x}},
$$

since $\bar{g}^{-1} \bar{h} \bar{g} \in G_{\bar{x}}$. Thus, $\mu_{\bar{g} \bar{x}}$ is left-invariant under the action of $G_{\bar{g} \bar{x}}$ on itself and must be a multiple of $d_{G_{\bar{g} \bar{x}}}$. That is, there is a constant $C_{\bar{g}}$ such that

$$
\int_{G_{\bar{x}}} F\left(\bar{g} h \bar{g}^{-1}\right) d_{G_{\bar{x}}} h=C_{\bar{g}} \int_{G_{\bar{g} \bar{x}}} F(h) d_{G_{\bar{g} \bar{x}}} h .
$$

Setting $F \equiv 1$, we find $C_{\bar{g}}=\left|G_{\bar{x}}\right| /\left|G_{\bar{g} \bar{x}}\right|=\Delta(\bar{g})$.

For any measure $\Phi$ on $G$, denote by $\mathcal{L}_{\Phi}$ the left convolution operator $F \mapsto \Phi * F$ on $G$, and by $\mathcal{R}_{\Phi}$ the right convolution operator $F \mapsto F * \Phi$, where

$\Phi * F(\bar{g})=\int_{G} F\left(g^{-1} \bar{g}\right) d \Phi(g) \quad$ and $\quad F * \Phi(\bar{g})=\int_{G} F\left(\bar{g} g^{-1}\right) \Delta\left(g^{-1}\right) d \Phi(g)$.

For all basic facts regarding convolution, see e.g. Hewitt and Ross [22].

With any transition operator on $X \times Y$ as in (2.2) and reference points $\bar{x} \in X, \bar{y} \in Y$, we associate a transition operator $\mathcal{K}=\mathcal{K}[K]$ on the group $G$ by

$$
\begin{aligned}
\mathcal{K}[K] & =\mathcal{T}_{\bar{x}} K \mathcal{S}_{\bar{y}}, \quad \text { that is, for } F: G \rightarrow \mathbb{R}, \\
\mathcal{K} F(g) & =\frac{1}{\left|G_{\bar{y}}\right|} \int_{Y} \int_{G_{\bar{y}}} F\left(g_{\bar{y}, y} h\right) d_{G_{\bar{y}}} h K(g \bar{x}, d y) .
\end{aligned}
$$


Denote by $\|\mathcal{K}\|_{p \rightarrow p}$ its norm on $L^{p}\left(G, d_{G}\right)$. Recall that for any function $F$ on $G$, the reflected function $\check{F}$ is defined by $\check{F}(g)=F\left(g^{-1}\right)$, and for any measure $\Phi$, the measure $\check{\Phi}$ is given by $\int_{G} F d \check{\Phi}=\int_{G} \check{F} d \Phi$.

(2.8) Proposition. (a)

$$
\|K\|_{p \rightarrow p}=\left(\frac{\left|G_{\bar{y}}\right|}{\left|G_{\bar{x}}\right|}\right)^{1 / p}\|\mathcal{K}\|_{p \rightarrow p} .
$$

(b) The operator $\mathcal{K}$ is a right convolution operator:

$$
\mathcal{K} F(\bar{g})=\int_{G} F\left(\bar{g} g^{-1}\right) \Delta\left(g^{-1}\right) d \Phi(g)
$$

where $\Phi=\Phi[K]$ is the measure on $G$ given by $\int_{G} F d \Phi=\mathcal{K}(\check{F} \check{\Delta})(\mathrm{id})$, that is, for a Borel set $B \subset G$, its measure is

$$
\Phi(B)=\frac{1}{\left|G_{\bar{y}}\right|} \int_{Y}\left|G_{y} \cap g_{\bar{y}, y} B\right|_{y} K(\bar{x}, d y),
$$

where $|\cdot|_{y}$ is the Haar measure on $G_{y}$.

Proof. (a) By Lemma 2.4(b) and the definition of $\mathcal{K}$, we have

$$
\left(\frac{\left|G_{\bar{y}}\right|}{\left|G_{\bar{x}}\right|}\right)^{1 / p}\|\mathcal{K}\|_{p \rightarrow p} \leq\|K\|_{p \rightarrow p}
$$

The reversed inequality follows from Lemma 2.4(a), which yields the identity $K=\mathcal{S}_{\bar{x}} \mathcal{K} \mathcal{T}_{\bar{y}}$.

(b) Group invariance of $K$ is the same as $\delta_{g} *(K f)=K\left(\delta_{g} * f\right)$ for every $g \in G$ and $f \in L^{p}\left(Y, d_{Y}\right)$. This and Lemma 2.4(c) imply that $\delta_{g} *(\mathcal{K} F)=\mathcal{K}\left(\delta_{g} * F\right)$ for every $g \in G$ and $F \in L^{p}\left(G, d_{G}\right)$. Therefore $\mathcal{K}$ is the right convolution operator on $G$ by the measure $\Phi$ given above.

Now, for any (non-negative) measure $\Phi$ on $G$, a routine computation shows that, with the measure $\widetilde{\Phi}_{p}$ given by

$$
\int_{G} F(g) d \widetilde{\Phi}_{p}(g)=\int_{G} \Delta(g)^{-1 / q} F\left(g^{-1}\right) d \Phi(g)
$$

one has

$$
\left\|\mathcal{R}_{\Phi}\right\|_{p \rightarrow p}=\left\|\mathcal{L}_{\widetilde{\Phi}_{p}}\right\|_{p \rightarrow p}
$$

compare e.g. with Hewitt and Ross [22, §20]. Furthermore, if $\Psi$ is a nonnegative measure on $G$ then $\left\|\mathcal{L}_{\Psi}\right\|_{p \rightarrow p} \leq \Psi(G)$, and when $G$ is amenable, 
this is an equality, see e.g. Reiter's book, 2nd edition: Reiter and Stegeman [30, Thm. 8.3.10]. In addition, if $\Psi$ is finite and $\operatorname{supp}(\Psi) \operatorname{supp}(\Psi)^{-1}$ generates $G$ as a closed group, then by Berg and Christensen [1], [2],

$$
\left\|\mathcal{L}_{\Psi}\right\|_{p \rightarrow p}=\int_{G} \Psi(g) d_{G} g \Longleftrightarrow G \text { is amenable. }
$$

(For $p \neq 2$, see the comments in Saloff-Coste and Woess [34].)

In the sequel, we shall write $\langle A\rangle$ for the subgroup of $G$ generated by $A \subset G$, and $\langle A\rangle^{-}$for its closure. Also, we shall write

$$
S[K]=S[K, \bar{x}, \bar{y}]=\{g \in G: g \bar{y} \in \operatorname{supp}(K(\bar{x}, \cdot))\} .
$$

This is the support of the measure $\check{\Phi}[K]$. The set $S[K]$ depends on the choice of $\bar{x}$ and $\bar{y}$, but $S[K] S[K]^{-1}$ is independent of $\bar{y}$ and depends on $\bar{x}$ only up to conjugation by an element of $G$.

Combining all these facts with Proposition 2.8, one immediately gets the following.

(2.12) Theorem. The norm $\sigma_{p}(K)$ of $K: L^{p}\left(Y, d_{Y}\right) \rightarrow L^{p}\left(X, d_{X}\right)$ satisfies

$$
\sigma_{p}(K) \leq a_{p}(K) \stackrel{\text { def }}{=} \int_{Y}\left(\frac{\left|G_{y}\right|}{\left|G_{\bar{x}}\right|}\right)^{1 / p} K(\bar{x}, d y) .
$$

Furthermore, this is an equality if and only if the subgroup $\left\langle S[K] S[K]^{-1}\right\rangle^{-}$ of $G$ is amenable; the "only if" requires finiteness of $a_{p}(K)$.

Proof. This follows from (2.8)-(2.10) by computing

$$
\widetilde{\Phi}_{p}(G)=\mathcal{K} \Delta^{-1 / p}(\mathrm{id})=\int_{Y}\left(\frac{\left|G_{y}\right|}{\left|G_{\bar{y}}\right|}\right)^{1 / p} K(\bar{x}, d y)=\left(\frac{\left|G_{\bar{y}}\right|}{\left|G_{\bar{x}}\right|}\right)^{-1 / p} a_{p}(K) .
$$

Note that by group invariance of $K$ and by Lemma 2.6(b), the number $a_{p}(K)$ does not depend on the choice of $\bar{x}$. In general, it might happen that $a_{p}(K)=\infty$ even when $K$ is stochastic. We might as well add the condition $a_{p}(K)<\infty$ to our assumptions. For example, this holds in the specific case when $K$ is stochastic and the measure $\Phi=\Phi[K]$ on $G$ is symmetric. Indeed, in this case,

$$
\int_{G} \Delta d \Phi=\int_{G} \Delta^{-1} d \Phi=1
$$

by Proposition 2.8(b), whence

$$
\Phi(G) \leq \frac{1}{2} \int_{G}\left(\Delta+\Delta^{-1}\right) d \Phi=1,
$$

so that Hölder's inequality implies

$$
a_{p}(K)=\int_{G} \Delta^{-1 / q} d \Phi \leq \Phi(G)^{1 / p}\left(\int_{G} \Delta^{-1} d \Phi\right)^{1 / q} \leq 1 .
$$


Next, we consider the adjoint operator $K^{*}$ of $K$. Formally, it is defined by the equation

$$
\int_{X} f(x) K f^{\prime}(x) d_{X} x=\int_{Y} K^{*} f(y) f^{\prime}(y) d_{Y} y .
$$

for $f \in \mathcal{C}_{00}(X)$ and $f^{\prime} \in \mathcal{C}_{00}(Y)$ (the respective spaces of continuous real functions with compact support). Local finiteness is needed here. (Note that $K^{*}$ does not have to be finite when $K$ is finite, while it will be locally finite when $K$ has this property.)

A priori, the way to compute $K^{*}$ is the following: we have $K=\mathcal{S}_{\bar{x}} \mathcal{K} \mathcal{T}_{\bar{y}}$. Straightforward computation yields $\mathcal{T}_{\bar{y}}^{*}=\left|G_{\bar{y}}\right| \mathcal{S}_{\bar{y}}$ and $\mathcal{S}_{\bar{x}}^{*}=\left|G_{\bar{x}}\right|^{-1} \mathcal{T}_{\bar{x}}$. Furthermore, as $\mathcal{K}=\mathcal{R}_{\Phi}$, where $\Phi=\Phi[K]$ is given by Proposition 2.8, we have $\mathcal{K}^{*}=\mathcal{R}_{\Phi^{*}}$, where the measure $\Phi^{*}=\Phi^{*}[K]$ is given by $d \Phi^{*}(g)=\Delta(g) d \check{\Phi}(g)$. Thus one gets

$$
K^{*}=\frac{\left|G_{\bar{y}}\right|}{\left|G_{\bar{x}}\right|} \mathcal{S}_{\bar{y}} \mathcal{R}_{\Phi^{*}} \mathcal{T}_{\bar{x}}
$$

From here one finds after some computation the following two formulas that a posteriori are easily verified.

(2.15) Proposition. For $f \in \mathcal{C}_{00}(X)$ and arbitrary $y_{0} \in Y$,

$$
\begin{aligned}
K^{*} f\left(y_{0}\right) & =\frac{1}{\left|G_{\bar{x}}\right|} \int_{Y} \int_{G_{y}} f\left(g_{y, y_{0}} h \bar{x}\right) d_{G_{y}} h K(\bar{x}, d y) \\
& =\frac{1}{\left|G_{\bar{x}}\right|} \int_{Y} \frac{\left|G_{y}\right|}{\left|G_{y_{0}}\right|} \int_{G_{y_{0}}} f\left(h g_{y, y_{0}} \bar{x}\right) d_{G_{y_{0}}} h K(\bar{x}, d y) .
\end{aligned}
$$

Proof. The identity between the two formulas follows immediately from Lemma 2.6(c+b). We now show that, when defining $K^{*} f$ in this way, the equation (2.13) does indeed hold:

$$
\begin{array}{rl}
\int_{Y} K^{*} & f\left(y_{0}\right) f^{\prime}\left(y_{0}\right) d_{Y} y_{0} \\
& =\frac{1}{\left|G_{\bar{x}}\right|} \int_{Y}\left(\int_{Y} \int_{G_{y}} f^{\prime}\left(g_{y, y_{0}} h y\right) f\left(g_{y, y_{0}} h \bar{x}\right) d_{G_{y}} h d_{Y} y_{0}\right) K(\bar{x}, d y) \\
& =\frac{1}{\left|G_{\bar{x}}\right|} \int_{Y}\left(\int_{G} f^{\prime}(g y) f(g \bar{x}) d_{G} g\right) K(\bar{x}, d y) \\
& =\frac{1}{\left|G_{\bar{x}}\right|} \int_{G} f(g \bar{x}) K\left(\delta_{g^{-1}} * f^{\prime}\right)(\bar{x}) d_{G} g \\
& =\frac{1}{\left|G_{\bar{x}}\right|} \int_{G} f(g \bar{x}) K f^{\prime}(g \bar{x}) d_{G} g=\int_{X} f(x) K f^{\prime}(x) d_{X} x
\end{array}
$$

In the second identity we have used that $y_{0}=g_{y, y_{0}} h y$ for all $h \in G_{y}$. Formula (2.1) was used in the second and in the last identity. 
(2.16) Lemma.

$$
a_{p}(K)=a_{q}\left(K^{*}\right)
$$

Proof. Let $\Phi=\Phi[K]$. Then one finds $\widetilde{\Phi^{*}}(G)=\int_{G} \Delta^{-1 / q} d \Phi=\widetilde{\Phi}_{p}(G)$. Now (2.14) yields

$$
a_{q}\left(K^{*}\right)=\frac{\left|G_{\bar{y}}\right|}{\left|G_{\bar{x}}\right|}\left(\frac{\left|G_{\bar{x}}\right|}{\left|G_{\bar{y}}\right|}\right)^{1 / q} \widetilde{\Phi^{*}}(G)=a_{p}(K) .
$$

After amenability, we next want to involve the question of unimodularity in our considerations. We define $b(K)=K(\bar{x}, Y)$, the total mass of $K$. It is of course independent of $\bar{x}$.

(2.17) Proposition. If $K$ satisfies (2.2) then

$$
a_{p}(K) \leq b(K)^{1 / q} b\left(K^{*}\right)^{1 / p} .
$$

Equality holds if and only if the modular function $\Delta$ of $G$ is constant on $S=S[K]$, or equivalently, if $\Delta \equiv 1$ on $\left\langle S S^{-1}\right\rangle^{-}$.

Assume furthermore that the set $S$ satisfies the condition

(i) there is $k>0$ such that $\int_{\left(S S^{-1}\right)^{k}} d_{G}>0$ (this holds, in particular, when $\left.\int_{S} d_{G}>0\right)$.

Then equality holds if and only if the group $\left\langle S S^{-1}\right\rangle$ (which is open-closed under this assumption) is unimodular.

Again, the "only if" requires finiteness of $b(K)^{1 / q} b\left(K^{*}\right)^{1 / p}$.

Proof. We have

$$
b\left(K^{*}\right)=\Phi * \mathbf{1}(\bar{y})=\int_{Y} \frac{\left|G_{y}\right|}{\left|G_{\bar{x}}\right|} K(\bar{x}, d y) .
$$

We now apply Hölder's inequality:

$$
\begin{aligned}
a_{p}(K) & =\int_{Y} 1 \cdot\left(\frac{\left|G_{y}\right|}{\left|G_{\bar{x}}\right|}\right)^{1 / p} K(\bar{x}, d y) \\
& \leq\left(\int_{Y} K(\bar{x}, d y)\right)^{1 / q}\left(\int_{Y} \frac{\left|G_{y}\right|}{\left|G_{\bar{x}}\right|} K(\bar{x}, d y)\right)^{1 / p} .
\end{aligned}
$$

Equality holds if and only if there is a constant $c_{0}$ such that $\left|G_{y}\right|=c_{0}$ for $K(\bar{x}, \cdot)$-almost every $y \in Y$. Equivalently, $\left|G_{g^{-1} \bar{y}}\right|=c_{0}$ for $\Phi[K]$-almost every $g \in S[K]$. Again equivalently, this means that there is $c>0$ such that $\Delta(g)=c$ for $\Phi[K]$-almost every $g \in S$. Since $\Delta$ is continuous, it must be constant on $S$. Since $\Delta(g h)=\Delta(g) \Delta(h)$, this is equivalent with $\Delta \equiv 1$ on $\left\langle S S^{-1}\right\rangle^{-}=\left(\bigcup_{n \geq 1}\left(S S^{-1}\right)^{n}\right)^{-}$. 
Suppose that (i) holds. Then $\left(S S^{-1}\right)^{2 k}$ contains an open set $V \neq \emptyset$, see e.g. Hewitt and Ross [22, Cor. 20.17]. Therefore, for each $n \geq 1$, we have

$$
\left(S S^{-1}\right)^{2 k+n} \supset V\left(S S^{-1}\right)^{n}
$$

and

$$
\left\langle S S^{-1}\right\rangle=\bigcup_{n \geq 1}\left(S S^{-1}\right)^{2 k+n} \supset V \bigcup_{n \geq 1}\left(S S^{-1}\right)^{n} \supset\left\langle S S^{-1}\right\rangle^{-}
$$

Thus, $\left\langle S S^{-1}\right\rangle$ is open and closed, and its modular function is the restriction of $\Delta$.

(2.21) Remark. Much of the above simplifies considerably when $K$ has a density, i.e., $K(x, d y)=k(x, y) d_{Y} y$, where $k(\cdot, \cdot)$ is a measurable function on $X \times Y$. In this case, the measure $\Phi[K]$ has the density

$$
\phi(g)=k\left(\bar{x}, g^{-1} \bar{y}\right) /\left|G_{\bar{y}}\right|
$$

with respect to the Haar measure $d_{G}$, and the adjoint operator $K^{*}$ has density $k^{*}(x, y)=k(y, x)$. We can always redefine $k(\cdot, \cdot)$, if necessary, such that the support of the measure $K(\bar{x}, \cdot)$ coincides with the support of the function $k(\bar{x}, \cdot)$ in $Y$. (The support of a function is the closure of the set where the function is non-zero.) Consequently, $S[K]=\operatorname{supp}(\phi)$ in the group $G$, and in the above results, we may replace $S[K]$ with $\operatorname{supp}(\phi)$.

To conclude this section, let $X, Y, Z$ be three homogeneous $G$-spaces, equipped with $G$-invariant measures $d_{X}, d_{Y}$ and $d_{Z}$, respectively, and transition operators $K$ from $X$ to $Y$ and $L$ from $Y$ to $Z$. We denote by $K L$ the transition operator from $X$ to $Z$ where

$$
K L(x, B)=\int_{Y} L(y, B) K(x, d y), \quad B \in \mathcal{B}(Z)
$$

Note that we say "transition operator from $X$ to $Y$ " because in our thinking, the expression $K(x, B), B \in \mathcal{B}(Y)$, describes a "rule" for going from $x \in X$ to $B \subset Y$. Of course, the corresponding linear operator goes the other way, taking functions on $Y$ to functions on $X$, and in that sense, $K L$ corresponds to first applying $L$ and then $K$. By inspection one obtains the following.

(2.22) Lemma. If both $K$ and $L$ satisfy (2.2), then

$$
\begin{gathered}
\Phi_{\bar{x}, \bar{z}}[K L]=\Phi_{\bar{y}, \bar{z}}[L] * \Phi_{\bar{x}, \bar{y}}[K] \\
a_{p}(K L)=a_{p}(K) a_{p}(L) \quad \text { and } \quad b(K L)=b(K) b(L) .
\end{gathered}
$$




\section{Transition operators on a homogeneous $G$-space}

In this section we consider the special case of $\S 2$ when $Y=X$, the group $G$ acts transitively on $X$, and $K$ is $G$-invariant (1.2). In all objects defined in $\S 2$, we shall set $\bar{y}=\bar{x}$.

We then have $S[K]=\{g \in G: g \bar{x} \in \operatorname{supp}(K(\bar{x}, \cdot))\}$. Besides the quantities $a_{p}(K), b(K), b\left(K^{*}\right)$ and the norm $\sigma_{p}(K)$, we also have the spectral radius $\rho_{p}(K)=\lim _{n} \sigma_{p}\left(K^{n}\right)^{1 / n}$, where $K^{n}$ is the $n$-th iterate of the operator $K$ on $X$.

The transition operator $K$ is called irreducible if for every $x \in X$ and every open subset $U$ of $X$, there is $n$ such that $K^{n}(x, U)>0$ for the $n$-th iterate $K^{n}$ of $K$. As a matter of fact, by transitivity it is enough to require this for $\bar{x}$ (and every open $U$ ) only. Equivalently, this means that the closed semigroup in $G$ generated by $S[K]$ coincides with $G$,

$$
\left(\bigcup_{n} S[K]^{n}\right)^{-}=G \text {. }
$$

In this case, it follows from Woess [42] that

$$
N(K)=\left(\bigcup_{n} S[K]^{n} S[K]^{-n}\right)^{-}
$$

is a normal subgroup of $G$ that either has finite index and hence is openclosed, or else $N(K)$ as well as each $S[K]^{n}$ is nowhere dense in $G$.

We now specialize the results of $\S 2$ to the transitive case. We have

$$
a_{p}(K)=\int_{X}\left(\frac{\left|G_{x}\right|}{\left|G_{\bar{x}}\right|}\right)^{1 / p} K(\bar{x}, d x),
$$

$b(K)=a_{\infty}(K)=K(x, X)$ for all $x$, and $b\left(K^{*}\right)=a_{1}(K)$. We remark that $d_{X}$ is an invariant measure for $K$ if and only if $K^{*}$ is stochastic, i.e., $K^{*}(\cdot, K) \equiv 1$, see $(2.13)$. (This fact does not rely on group invariance.)

(3.2) Theorem. Suppose that $K$ is $G$-invariant, and that $G$ acts transitively on $X$. Then the following statements are true.

$$
\sigma_{p}(K) \leq a_{p}(K),
$$

and equality holds if and only if the subgroup $\left\langle S[K] S[K]^{-1}\right\rangle^{-}$of $G$ is amenable.

$$
\rho_{p}(K) \leq a_{p}(K),
$$

and equality holds if and only if the subgroup $\langle S[K]\rangle^{-}$of $G$ is amenable.

$$
a_{p}(K) \leq b\left(K^{*}\right)^{1 / p}
$$

and equality holds if and only if $\Delta \equiv 1$ on $\left\langle S[K] S[K]^{-1}\right\rangle^{-}$. If $K$ is irreducible and $N(K)$ is open, then equality holds if and only if $G$ is unimodular.

In all three statements, the "only if" requires finiteness of the respective upper bound. 
Proof. Statement (a) is Corollary 2.23, specified to the one-orbit-case.

For the inequality in (b), we use (a) and Lemma 2.22, which imply $\sigma_{p}\left(K^{n}\right) \leq a_{p}(K)^{n}$. For the "if and only if" in (b), we use the result of Berg and Christensen [1], [2], by which the $L^{p}$-spectral radius of the left convolution operator with $\widetilde{\Phi}_{p}$ coincides with $\widetilde{\Phi}_{p}(G)$ if and only if the closed group generated by $\operatorname{supp}\left(\widetilde{\Phi}_{p}\right)$ is amenable.

The first part of (c) is Proposition 2.17.

The addendum regarding the irreducible case in statement (c) is seen as follows. If equality holds in (c), then it will also hold for $K^{n}$ in the place of $K$, for any $n \geq 1$. Thus, $\Delta \equiv 1$ on $S[K]^{n} S[K]^{-n}$, and (since $\Delta$ is continuous) $\Delta(g)=1$ for every $g \in N(K)$. Now $N(K)$ has finite index (as a matter of fact $G / N(K)$ is cyclic), so that $\Delta \equiv 1$ on the whole of $G$.

Note that condition (i) of Proposition 2.17, or

(ii) there is $k$ such that $S[K]^{k} S[K]^{-k}$ has positive Haar measure, assure the validity of the "if and only if" in statement (c) of the last theorem.

(3.3) Corollary. Let $X, K, G$ as in Theorem (3.2). If the group $\langle S[K]\rangle^{-}$ is amenable then $\sigma_{p}(K)=a_{p}(K)=\rho_{p}(K)$. In particular, in this case, norm and spectral radius coincide on $L^{p}$ for every $p \in(1, \infty)$.

In the irreducible, homogeneous case, when $K(\cdot, X)<\infty$, besides the

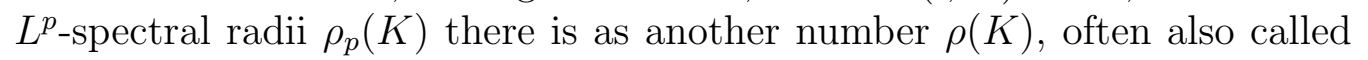
spectral radius, defined by

$$
\limsup _{n} K^{n} f(x)^{1 / n}=\rho(K) \quad \text { for all } f \in \mathcal{C}_{00}^{+}(X), x \in X
$$

(As usual, $\mathcal{C}_{00}$ denotes the space of compactly supported, continuous functions.) That $\rho(K)$ is independent of $f$ follows from the corresponding theorem regarding convolution powers on locally compact groups, see Guivarc'h [19]. Indeed, this reference shows that the number $\lim \sup _{n} \mathcal{K}^{n} F(g)^{1 / n}$ is independent of $F \in \mathcal{C}_{00}^{+}(G)$. As $\mathcal{T}_{\bar{x}} K^{n}=\mathcal{K}^{n} \mathcal{T}_{\bar{x}}$, this carries over to the operator $K$ on $X$. In general, $\rho(K) \leq \rho_{2}(K)$, but when $K$ is self-adjoint with respect to $d_{X}$, this is an equality, see Berg and Christensen [2]. ${ }^{2}$

As a "by-product", we obtain a proof of Theorem II $(\S 1)$ that does not rely on any specific ingredient from differential geometry.

\footnotetext{
${ }^{2}$ Some translation is necessary here, since Berg and Christensen deal with left convolution operators with respect to left Haar measure. The point is the following: since we know that the upper limit is independent of $F \in \mathcal{C}_{00}^{+}(G)$, we see that $\rho(\mathcal{K})=\rho\left(\mathcal{L}_{\widetilde{\Phi}_{2}}\right)$, where $\widetilde{\Phi}_{2}$ is as defined before (2.9). Also, since $K$ is self-adjoint the measure $\widetilde{\Phi}_{2}$ is symmetric, as required.
} 
(3.5) Corollary. Let $\mathcal{M}$ be a connected non-compact Riemannian manifold which is homogeneous in the sense that its group $\operatorname{Iso}(\mathcal{M})$ of isometries acts transitively.

Then 0 is in the $L^{2}$-spectrum of the Laplacian of $\mathcal{M}$ (or equivalently, the heat kernel $k_{t}(\cdot, \cdot)$ on $\mathcal{M}$ has sub-exponential decay as $\left.t \rightarrow \infty\right)$ if and only if some (equivalently, every) closed, transitive subgroup of $\operatorname{Iso}(\mathcal{M})$ is both amenable and unimodular.

Proof. The invariant measure $d_{X}=d_{\mathcal{M}}$ is the Riemannian volume element. It is well known - see e.g. Davies [11, Prop. 12] - that the heat kernel has exponential decay if and only if $\rho_{2}\left(K_{1}\right)<1$, where $K_{1}$ is given by $K_{1}(x, d y)=k_{1}(x, y) d y$. This transition operator is stochastic and selfadjoint, and $S\left[K_{1}\right]=G$. Theorem 3.2 applies.

As announced, we shall extend this to the co-compact case in the next sections. Let us now give a simple example to illustrate how our method may be used to compute norms and spectral radii explicitly.

(3.6) Example. Let $X=\mathbb{H}=\{z=x+\mathfrak{i} y \in \mathbb{C}: y>0\}$ be the hyperbolic plane with length element $d s=|d z| / \Im(z)$ and area element $d_{\mathbb{H}} z=d z / \Im(z)^{2}$. Fix $r>0$ and consider the ball $B(z, r)$ with radius $r$ and centre $z$ in the hyperbolic metric. Let $V(r)=2 \pi \cosh r$ be its hyperbolic area, and define a transition operator $K=K_{(r)}$ by

$$
K f(z)=\frac{1}{V(r)} \int_{B(z, r)} f(z) d_{\mathbb{H}} z .
$$

As mentioned in the Introduction, this corresponds to a random walk, where at each step, the walker - given that his acutal position is $z$ - moves to a random point that is chosen according to hyperbolic equidistribution in $B(z, r)$. This random walk is symmetric ( $d_{\mathbb{H}}$-reversible), and $K$ is clearly irreducible.

The operator is invariant under the whole isometry group of $\mathbb{H}$, which is non-amenable. We prefer to work with a smaller group of isometries, namely the affine group

$$
G=\{z \mapsto a z+b: a>0, b \in \mathbb{R}\} .
$$

It acts by isometries, fixed-point-freely and transitively on $\mathbb{H}$ and preserves $d_{\mathbb{H}}$. The left Haar measure is $d_{G}=a^{-2} d a d b$, where $d a$ and $d b$ are Lebesgue measure on $\mathbb{R}_{+}$, resp. $\mathbb{R}$. The modular function is $\Delta(g)=1 / a$, when $g=(a, b)$. We chose the complex unit $\mathfrak{i}$ as our reference point in $\mathbb{H}$.

If $z=x+\mathfrak{i} y \in \mathbb{H}$ then $z=g \mathfrak{i}$, where $g=(y, x)$. Therefore, by Lemma 2.6, $\left|G_{z}\right| /\left|G_{\mathrm{i}}\right|=y$. Note that all point stabilizers are trivial, but formula (2.1) 
forces $\left|G_{z}\right|=\Im(z)$. Since the affine group is amenable, we can compute by Theorem 3.2

$$
\rho_{p}(K)=\sigma_{p}(K)=a_{p}(K)=\frac{1}{V(r)} \int_{B(\mathfrak{i}, r)} y^{-2+1 / p} d x d y .
$$

Passing from the upper half plane model of $\mathbb{H}$ to the unit disk model via the map $z \mapsto w=(\mathfrak{i} z+1) /(z+\mathfrak{i})$, the volume element becomes $d w=$ $4\left(1-|w|^{2}\right)^{-2} d x d y$ for $w=x+\mathfrak{i} y$, and $B(\mathfrak{i}, r)$ transforms to $B(0, r)$ in the transformed metric. Writing $P(w, \xi)$ for the Poisson kernel in the unit disk, the formula becomes (using rotation invariance in the second equality, with $d \xi$ the normalized Lebesgue measure on the unit circle $\mathbb{S}^{1}$ )

$$
\begin{aligned}
\rho_{p}(K) & =\frac{1}{V(r)} \int_{B(0, r)} P(w, \mathfrak{i})^{1 / p} d w=\frac{1}{V(r)} \int_{B(0, r)} \int_{\mathbb{S}^{1}} P(w, \xi)^{1 / p} d \xi d w \\
& =\frac{1}{V(r)} \int_{B(0, r)} \varphi_{\alpha}(w) d w
\end{aligned}
$$

where $\alpha=\frac{1}{2}-\frac{1}{p}$ and

$$
\varphi_{\alpha}(w)=\int_{\mathbb{S}^{1}} P(w, \xi)^{\alpha+1 / 2} d \xi
$$

is a spherical function. It depends only on

$$
t=d(w, 0)=\log \frac{1+|w|}{1-|w|}
$$

(hyperbolic distance $d(w, 0)$ in the unit disk), i.e., $\varphi_{\alpha}(w)=\phi_{\alpha}(t)$. Thus, we get

$$
\rho_{p}(K)=\frac{1}{\cosh r-1} \int_{0}^{r} \phi_{\alpha}(t) \sinh t d t .
$$

An analogous formula holds for arbitrary isotropic (rotation invariant) transition operators. For example, if $S(z, r)$ denotes the hyperbolic sphere (circle) of radius $r$ centered at $z$, and $d_{l} z$ is the hyperbolic length element, then -writing $\ell(r)$ for the length of $S(z, r)$ - we can compute the spectral radii of the averaging operator over spheres $L=L_{(r)}$,

$$
L f(z)=\frac{1}{\ell(r)} \int_{S(z, r)} f d_{l}, \quad \rho_{p}(L)=\phi_{\alpha}(r), \quad \alpha=\frac{1}{2}-\frac{1}{p} .
$$

The value $\rho_{2}(L)$ has been computed by a different method by Żuk [43], using amenability and Følner sets. The formulas can of course also be obtained within the well-known framework of radial harmonic analysis on $\mathbb{H}$, but the present method is more elementary or at least more direct. 


\section{Decomposition of measures and transition operators on co-compact $G$-spaces}

In this section, we finally arrive at the general situation proposed by the title of this paper. That is, we suppose that the group $G$ acts properly (not necessarily isometrically) on $X$, that closed balls in $X$ are compact, and that the factor space $I=G \backslash X$ is compact. On $X$, we shall consider a transition operator $K$ that satisfies (1.2). We write $X_{i}, i \in I$, for the orbits of $G$ on $X$.

Let $d_{X}$ be a $G$-invariant measure on $X$. Each $X_{i}$ is a homogeneous $G$ space carrying a $G$-invariant measure $d_{X_{i}}$. We assume that the latter are normalized so that

$$
x \mapsto\left|G_{x}\right| \quad \text { is measurable and locally bounded on } X \text {, }
$$

and that there exists a measure $\lambda$ on $I$, supported on the whole of $I$, such that for $f \in \mathcal{C}_{00}(X)$, writing $f_{i}=\mathcal{P}_{i} f$ for the restriction of $f$ to $X_{i}$,

$$
\int_{X} f(x) d_{X} x=\int_{I} \int_{X_{i}} f_{i}(x) d_{X_{i}} x d \lambda(i) .
$$

We then speak of a measurable decomposition of $d_{X}$. If, in addition, $x \mapsto$ $\left|G_{x}\right|$ is continuous on $X$ (it is continuous on each $X_{i}$ ), then we speak of a continuous decomposition of $d_{X}$.

Combining (4.1) with Lemma 2.6, it is easy to see that the function

$$
f^{b}(i)=\int_{X_{i}} f_{i}(x) d_{X_{i}} x
$$

is measurable and bounded on $I$ for every $f \in \mathcal{C}_{00}(X)$, continuous on $I$ when $x \mapsto\left|G_{x}\right|$ is continuous on $X$. If $f$ is an arbitrary $d_{X}$-integrable function on $X$, then one sees from Bourbaki [3, Chap. VII, $\S 2$, Prop. 5] that the function $f^{b}(i)$, defined as in (4.3), exists and is finite for $\lambda$-almost every $i \in I$, and that $\int_{X} f(x) d_{X} x=\int_{I} f^{b}(i) d \lambda(i)$ precisely as in (4.2). ${ }^{3}$

In the Appendix ( $(9)$, we show that (i) one can use (4.2) to actually construct invariant measures on $X$, and (ii) for any $G$-invariant measure $d_{X}$ there exists a measure $\lambda$ on $I$ such that $d_{X}$ has a continuous decomposition with respect to $\lambda$ (see Lemma 9.3 and Proposition 9.5).

\footnotetext{
${ }^{3}$ Again, some translation is necessary in the use of the cited chapter of Bourbaki. There, the group - called $H$ - acts on $X$ from the right, while left Haar measure is used. In our case, this would require use of right Haar measure. Thus, to achieve compatibility with Bourbaki's notation up to the exchange between left and right, what we denote by $f^{b}$ should be written $\widetilde{f}^{b}$, where $\widetilde{f}(x)=f(x) /\left|G_{x}\right|$.
} 
Let us now consider the $G$-invariant transition operator $K$. We want to relate properties of $G$ (amenability, unimodularity) with the computation of the norm of $K$ on $L^{p}\left(X, d_{X}\right)$ and more generally on $L^{p}(X, \mu)$. In order to apply the results of $\S 2$ to the present situation, besides the measurable decomposition (4.2) of $d_{X}$, we need a decomposition of $K$, that is, a family $K_{j}(\cdot, \cdot), j \in I$, of transition operators from $X$ to $X_{j}$, with the following properties

$$
\begin{aligned}
K_{j}(g x, g B)=K_{j}(x, B) & \text { for all } x \in X, B \in \mathcal{B}\left(X_{j}\right), g \in G \\
(j, x) \mapsto K_{j}\left(x, B \cap X_{j}\right) & \text { is measurable for every } B \in \mathcal{B}(X)
\end{aligned}
$$

and, for $d_{X}$-almost every $x \in X$,

$$
K(x, B)=\int_{I} K_{j}\left(x, B \cap X_{j}\right) d \lambda(j) \quad \text { for all } B \in \mathcal{B}(X) .
$$

Such a decomposition will not always exist. In the Appendix (Theorem 9.8), we give a necessary and sufficient existence condition in the case when $K$ is finite. The decomposition is of course straightforward when $K$ has a density with respect to $d_{X}$, see $\S 7$.

Now let us suppose to have a decomposition (4.2) of $d_{X}$ and an associated decomposition (4.4) of $K$. For $i, j \in I$, we define $K_{i, j}$ as the restriction of the operator $K_{j}$ to $X_{i}$, that is, $K_{i, j}=\mathcal{P}_{i} K_{j}$, or equivalently, for every $x \in X_{i}$ and $B \in \mathcal{B}\left(X_{j}\right)$, we get $K_{i, j}(x, B)=K_{j}(x, B)$.

With this definition, for all $f \in \mathcal{C}_{00}(X)$ and almost all $x \in X$, we have

$$
K f(x)=\int_{I} K_{i, j} f_{j}(x) d \lambda(j) \quad \text { if } \quad x \in X_{i} .
$$

In particular, for all $f, f^{\prime} \in \mathcal{C}_{00}(X)$, we have

$$
\int_{X} f^{\prime}(x) K f(x) d_{X} x=\int_{I} \int_{I} \int_{X_{i}} f_{i}^{\prime}(x) K_{i, j} f_{j}(x) d_{X_{i}} x d \lambda(j) d \lambda(i) .
$$

Using the notation of $\S 2$, for $p \in(1, \infty)$, define

$$
\sigma_{p}(i, j)=\sigma_{p}\left(K_{i, j}, d_{X_{i}}, d_{X_{j}}\right) .
$$

This is the norm of $K_{i, j}$ as an operator from $L^{p}\left(X_{j}, d_{X_{j}}\right)$ to $L^{p}\left(X_{i}, d_{X_{i}}\right)$.

(4.5) Lemma. The mapping $(i, j) \mapsto \sigma_{p}(i, j)$ on $I \times I$ is measurable.

Proof. Let $f \in \mathcal{C}_{00}(X)$ such that $\mathcal{P}_{j} f$ is non-zero. Then, by (4.3) and (4.4), the mapping

$$
(i, j) \mapsto\left\|K_{i, j} \mathcal{P}_{j} f\right\|^{p} /\left\|\mathcal{P}_{j} f\right\|^{p}=\int_{X_{i}}\left|K_{j} f_{j}(x)\right|^{p} d_{X_{i}} x / \int_{X_{j}}\left|f_{j}(y)\right|^{p} d_{X_{j}} y
$$

is measurable on $I \times I$. 
Now, $\mathcal{P}_{j}: \mathcal{C}_{00}(X) \rightarrow \mathcal{C}_{00}\left(X_{j}\right)$ is surjective. This follows from the TietzeUrysohn Extension Theorem, see e.g. Copson [9, p. 106]. Thus, the image of $\mathcal{C}^{0}$ (defined as above) under $\mathcal{P}_{j}$ is dense in $\mathcal{C}_{00}\left(X_{j}\right)$. Therefore $(i, j) \mapsto \sigma_{p}(i, j)$ is the point-wise supremum of a countable family of measurable functions.

(4.6) Lemma. Let $K$ and $L$ be two $G$-invariant transition operators on $X$ for which the product transition operator $K L$ is defined. If both $K$ and $L$ have a decomposition (4.4), then this is also true for $K L$, and

$$
(K L)_{i, j}=\int_{I} K_{i, i^{\prime}} L_{i^{\prime}, j} d \lambda\left(i^{\prime}\right) .
$$

Furthermore,

$$
\sigma_{p}\left((K L)_{i, j}, d_{X_{i}}, d_{X_{j}}\right) \leq \int_{I} \sigma_{p}\left(K_{i, i^{\prime}}, d_{X_{i}}, d_{X_{i^{\prime}}}\right) \sigma_{p}\left(L_{i^{\prime}, j}, d_{X_{i^{\prime}}}, d_{X_{j}}\right) d \lambda\left(i^{\prime}\right) .
$$

Proof. The first statement is straightforward, using Fubini's theorem. For the second statement, take $f \in \mathcal{C}_{00}\left(X_{j}\right)$ and $f^{\prime} \in \mathcal{C}_{00}\left(X_{i}\right)$, and apply Hölder's inequality to get

$$
\begin{aligned}
\int_{X_{i}} f^{\prime}(x) & (K L)_{i, j} f(x) d_{X_{i}} x \leq \int_{I}\left\|f^{\prime}\right\|_{q}\left\|K_{i, i^{\prime}} L_{i^{\prime}, j} f\right\|_{p} d \lambda\left(i^{\prime}\right) \\
& \leq\left\|f^{\prime}\right\|_{q}\|f\|_{p} \int_{I} \sigma_{p}\left(K_{i, i^{\prime}}, d_{X_{i}}, d_{X_{i^{\prime}}}\right) \sigma_{p}\left(L_{i^{\prime}, j}, d_{X_{i^{\prime}}}, d_{X_{j}}\right) d \lambda\left(i^{\prime}\right) .
\end{aligned}
$$

The following is a straightforward exercise, using only (2.13).

(4.7) Lemma. If $K$ has a decomposition (4.4) then so does the adjoint $K^{*}$, and we have $\left(K^{*}\right)_{i, j}=\left(K_{j, i}\right)^{*}$.

We shall set $K_{i, j}^{*}=\left(K^{*}\right)_{i, j}$.

\section{General results regarding norms, spectral radii, ame- nability and unimodularity}

According to Parthasarathy [27, Th. I.4.2], we can find a measurable section, that is, a set $\left\{\bar{x}_{i}: i \in I\right\} \in \mathcal{B}(X)$ that is contained in the support of our normalizing function $\bar{f}$, such that $\bar{x}_{i} \in X_{i}$ for each $i$ and $i \mapsto \bar{x}_{i}$ is one-to-one and measurable. From (4.4) it follows that $(i, j) \mapsto a_{p}(i, j)$ is measurable, where $a_{p}(i, j)=a_{p}\left(K_{i, j}\right)$ as defined in Theorem 2.12. We can now consider the transition operators $\Sigma_{p}=\Sigma_{p}[K]$ and $\mathfrak{A}_{p}=\mathfrak{A}_{p}[K]$ on $I$, defined by

$$
\Sigma_{p}(i, A)=\int_{A} \sigma_{p}(i, j) d \lambda(j) \quad \text { and } \quad \mathfrak{A}_{p}(i, A)=\int_{A} a_{p}(i, j) d \lambda(j) .
$$


Let

$$
S=\left(\bigcup_{i, j \in I} S\left[K_{i, j}\right]\right)^{-},
$$

where $S\left[K_{i, j}\right]$ is defined as in (2.11) with respect to the reference points $\bar{x}_{i}$ and $\bar{x}_{j}$.

(5.3) Theorem. Suppose that the transition operator $K$ on $X$ is $G$ invariant, that $I=G \backslash X$ is compact, and that we have a measurable decomposition of $d_{X}$ on $X$ as in (4.2) and a decomposition of $K$ as in (4.4). Then the norm $\sigma_{p}(K)$ of $K$ on $L^{p}\left(X, d_{X}\right)$ satisfies

$$
\sigma_{p}(K) \leq \sigma_{p}\left(\Sigma_{p}[K]\right) \leq \sigma_{p}\left(\mathfrak{A}_{p}[K]\right) .
$$

Furthermore, if the group $\left\langle S S^{-1}\right\rangle^{-}$is amenable, then

$$
\sigma_{p}(K)=\sigma_{p}\left(\mathfrak{A}_{p}[K]\right) .
$$

Analogously, the spectral radius $\rho_{p}(K)$ of $K$ satisfies

$$
\rho_{p}(K) \leq \rho_{p}\left(\Sigma_{p}[K]\right) \leq \rho_{p}\left(\mathfrak{A}_{p}[K]\right),
$$

and if the group $\langle S\rangle^{-}$is amenable, then

$$
\rho_{p}(K)=\rho_{p}\left(\mathfrak{A}_{p}[K]\right) .
$$

Proof. The second of the two inequalities in (5.3.i) follows from Theorem 2.12. To prove the first, let $f, f^{\prime} \in \mathcal{C}_{00}(X)$. Write $\Sigma_{p}$ for $\Sigma_{p}[K]$ and set $u(i)=\left\|f_{i}\right\|_{p}$, the norm of $f_{i}=\mathcal{P}_{i} f$ in $L^{p}\left(X_{i}, d_{X_{i}}\right)$, and analogously $v(i)=\left\|f_{j}^{\prime}\right\|_{q}$. Then, applying twice Hölder's inequality,

$$
\begin{aligned}
\int_{X} f^{\prime}(x) K f(x) d_{X} x & =\int_{I} \int_{I} \int_{X_{i}} f_{i}^{\prime}(x) K_{i, j} f_{j}(x) d_{X_{i}} x, d \lambda(j) d \lambda(i) \\
& \leq \int_{I} v(i) \int_{I} \sigma_{p}(i, j) u(j) d \lambda(j) d \lambda(i) \\
& =\int_{I} v(i) \Sigma_{p} u(i) d \lambda(i) \leq \sigma_{p}\left(\Sigma_{p}\right)\left\|f^{\prime}\right\|_{q}\|f\|_{p}
\end{aligned}
$$

since $\|u\|_{p}=\|f\|_{p}$, where the first norm is in $L^{p}(I, d \lambda)$ and the second in $L^{p}\left(X, d_{X}\right)$, and analogously, $\|v\|_{q}=\left\|f^{\prime}\right\|_{q}$. This implies that $\sigma_{p}(K) \leq$ $\sigma_{p}\left(\Sigma_{p}[K]\right)$.

In order to prove the equality in (5.3.ii) when $\left\langle S S^{-1}\right\rangle^{-}$is amenable, note that for computing norms we can assume without loss of generality 
that (4.4.iii) holds for all $x \in X$. We use the Borel cross section $\left\{\bar{x}_{i}: i \in I\right\}$ from above. Recall from $\S 2$ the operators $\mathcal{S}_{\bar{x}_{i}}: L^{p}\left(G, d_{G}\right) \rightarrow L^{p}\left(X_{i}, d_{X_{i}}\right)$ and $\mathcal{T}_{\bar{x}_{i}}: L^{p}\left(X_{i}, d_{X_{i}}\right) \rightarrow L^{p}\left(G, d_{G}\right)$. Now let $u, v \in \mathcal{C}^{+}(I)$ (the cone of nonnegative continuous functions) such that $\|u\|_{p}=\|v\|_{q}=1$ with respect to the measure $\lambda$ on $I$. Define operators $\mathcal{T}_{v, q}: L^{p}\left(X, d_{X}\right) \rightarrow L^{p}\left(G, d_{G}\right)$ and $\mathcal{S}_{u, p}: L^{p}\left(G, d_{G}\right) \rightarrow L^{p}\left(X, d_{X}\right)$ by

$$
\begin{aligned}
\mathcal{T}_{v, p} f(g) & =\int_{I} v(i)\left|G_{\bar{x}_{i}}\right|^{-1 / p} \mathcal{T}_{\bar{x}_{i}} \mathcal{P}_{i} f(g) d \lambda(i) \quad \text { and } \\
\mathcal{S}_{u, p} F(x) & =u(i)\left|G_{\bar{x}_{i}}\right|^{1 / p} \mathcal{S}_{\bar{x}_{i}} F(x), \quad \text { if } x \in X_{i} .
\end{aligned}
$$

The property (4.4.ii) is crucial for this definition. It is straightforward that both operators have norm $\leq 1$. We finally introduce the operator

$$
\mathcal{K}=\mathcal{K}_{u, v, p} \stackrel{\text { def }}{=} \mathcal{T}_{v, p} K \mathcal{S}_{u, p}: L^{p}\left(G, d_{G}\right) \rightarrow L^{p}\left(G, d_{G}\right) .
$$

For $F \in L^{p}\left(G, d_{G}\right)$, we obtain

$$
\mathcal{K} F(g)=\int_{I} \int_{I} v(i) u(j)\left(\frac{\left|G_{\bar{x}_{j}}\right|}{\left|G_{\bar{x}_{i}}\right|}\right)^{1 / p} \mathcal{K}_{i, j} F(g) d \lambda(i) d \lambda(j),
$$

where $\mathcal{K}_{i, j}=\mathcal{K}\left[K_{i, j}\right]$, as defined in (2.7) with respect to the reference points $\bar{x}_{i}$ and $\bar{x}_{j}$. Since $\delta_{g} *(\mathcal{K} F)=\mathcal{K}\left(\delta_{g} * F\right)$ for every $g \in G$, the operator $\mathcal{K}$ is a right convolution operator. The support of the associated measure on $G$ is contained in $S$. Therefore (2.8)-(2.10) imply that

$$
\begin{aligned}
\sigma_{p}\left(\mathcal{K}, d_{G}\right) & =\mathcal{K} \Delta^{-1 / p}(\mathrm{id}) \\
& =\int_{I} \int_{I} v(i) u(j)\left(\frac{\left|G_{\bar{x}_{j}}\right|}{\left|G_{\bar{x}_{i}}\right|}\right)^{1 / p} \mathcal{K}_{i, j} \Delta^{-1 / p}(\mathrm{id}) d \lambda(i) d \lambda(j) \\
& =\int_{I} v(i) \int_{I} a_{p}(i, j) u(j) d \lambda(i) d \lambda(j),
\end{aligned}
$$

compare with Theorem 2.12 and its proof. Since $\sigma_{p}\left(\mathcal{K}_{u, v, p}, d_{G}\right) \leq \sigma_{p}\left(K, d_{X}\right)$, we get

$$
\int_{I} v(i) \mathfrak{A}_{p} u(i) d \lambda(i) \leq \sigma_{p}(K)
$$

for all $u, v \in \mathcal{C}^{+}(I)$ with $\|u\|_{p}=\|v\|_{q}=1$. As all our operators preserve non-negativity, the proposed equality follows.

We next prove (5.3.iii). Lemmas 4.6 and 2.22 imply that for $h \in \mathcal{C}^{+}(I)$,

$$
\Sigma_{p}\left[K^{n}\right] h \leq\left(\Sigma_{p}[K]\right)^{n} h \quad \text { and } \quad \mathfrak{A}_{p}\left[K^{n}\right] h=\left(\mathfrak{A}_{p}[K]\right)^{n} h
$$


where the powers of the respective transition operators are taken on $X$ on the left and on $I$ on the right hand sides. Since all our transition operators are non-negative (i.e., they preserve the respective cones of non-negative functions), this together with (5.3.i) yields

$$
\sigma_{p}\left(K^{n}\right) \leq \sigma_{p}\left(\Sigma_{p}[K]^{n}\right) \leq \sigma_{p}\left(\mathfrak{A}_{p}[K]^{n}\right)
$$

for every $n$. Taking $n$-th roots and passing to the limit, we obtain the proposed inequalities for the spectral radii.

Finally, regarding (5.3.iv), if $\langle S\rangle^{-}$is amenable, then we can apply (5.3.ii) to every power $K^{n}$ of $K$. Again, passing to the limit of the $n$-th roots, we get the proposed equality.

Formulas (5.3.ii) and (5.3.iv) show that in the amenable case, we can reduce the computation of norms and spectral radii of $K$ to that of suitable transition operators on the compact space $I$. This will be useful for concrete calculations; an example will be given at the end. We shall also study the question when the equalities (5.3.ii) and (5.3.iv) imply amenability of the group. This will require some additional assumptions, see $\S 5$ and $\S 6$, and we first consider the analogues of the bounds of Proposition 2.17.

Consider the numbers

$$
\begin{aligned}
b(i, j) & =b\left(K_{i, j}\right)=\int_{X_{j}} K_{i, j}\left(\bar{x}_{i}, d y\right) \text { and } \\
b_{*}(i, j)=b\left(K_{i, j}^{*}\right) & =\int_{X_{i}} \frac{\left|G_{x}\right|}{\left|G_{\bar{x}_{j}}\right|} K_{j, i}\left(\bar{x}_{j}, d x\right)
\end{aligned}
$$

and the associated transition operators $\mathfrak{B}=\mathfrak{B}[K]$ and $\mathfrak{B}_{*}=\mathfrak{B}_{*}[K]=$ $\mathfrak{B}\left[K^{*}\right]$ on $I$ given by

$$
\mathfrak{B}(i, A)=\int_{A} b(i, j) d \lambda(j) \quad \text { and } \quad \mathfrak{B}_{*}(i, A)=\int_{A} b_{*}(i, j) d \lambda(j),
$$

where $A \in \mathcal{B}(I)$.

(5.5) Proposition. Under the assumptions of Theorem 5.3, we have

$$
\begin{gathered}
\sigma_{p}\left(\mathfrak{A}_{p}[K]\right) \leq \sigma_{p}(\mathfrak{B}[K])^{1 / q} \sigma_{q}\left(\mathfrak{B}_{*}[K]\right)^{1 / p} \text { and } \\
\rho_{p}\left(\mathfrak{A}_{p}[K]\right) \leq \rho_{p}(\mathfrak{B}[K])^{1 / q} \rho_{q}\left(\mathfrak{B}_{*}[K]\right)^{1 / p}
\end{gathered}
$$

Furthermore, if $G$ is unimodular, then

$$
\rho_{p}\left(\mathfrak{A}_{p}[K]\right)=\rho_{p}(\mathfrak{B}[K])=\rho_{q}\left(\mathfrak{B}_{*}[K]\right),
$$

so that (5.5.ii) becomes an equality. 
Proof. Let $u, v \in \mathcal{C}^{+}(I)$ with $\|u\|_{p}=\|v\|_{q}=1$ (norms with respect to $\lambda$ ). By Proposition 2.17, $a_{p}(i, j) \leq b(i, j)^{1 / q} b_{*}(j, i)^{1 / p}$. This and Hölder's inequality imply

$$
\begin{aligned}
\int_{I} v(i) \mathfrak{A}_{p} u(i) d \lambda(i) & \leq \int_{I} \int_{I}(v(i) b(i, j) u(j))^{1 / q}\left(u(j) b_{*}(j, i) v(i)\right)^{1 / p} d \lambda(i) d \lambda(j) \\
& \leq\left(\int_{I} v(i) \mathfrak{B} u(i) d \lambda(i)\right)^{1 / q}\left(\int_{I} u(j) \mathfrak{B}_{*} v(j) d \lambda(j)\right)^{1 / p} \\
& \leq \sigma_{p}(\mathfrak{B})^{1 / q} \sigma_{q}\left(\mathfrak{B}_{*}\right)^{1 / p} .
\end{aligned}
$$

This yields the first inequality. The second follows, since Lemmas 4.6 and 2.22 imply that $\mathfrak{B}[K]^{n}=\mathfrak{B}\left[K^{n}\right]$ and $\mathfrak{B}_{*}[K]^{n}=\mathfrak{B}_{*}\left[K^{n}\right]$. Assume unimodularity. Then the function $x \mapsto\left|G_{x}\right|$ is constant on each $X_{i}$. Thus, $\delta(i)=\left|G_{\bar{x}_{i}}\right|$ defines a continuous, strictly positive function on $I$. We find that $a_{p}(i, j)=\delta(i)^{-1 / p} b(i, j) \delta(j)^{1 / p}$ and $b_{*}(i, j)=\delta(j)^{-1} b(j, i) \delta(i)$. Therefore (5.5.iii) holds by a basic exercise. (Note that the analogue of (5.5.iii) does not necessarily hold for the norms in the place of the spectral radii.)

(5.6) Remark. For the main results of this section, co-compactness is not essential. Suppose that $X$ is a proper metric space, $G$ a locally compact group that acts properly on $X$, and let $I=G \backslash X$ (not necessarily compact). Consider a $G$-invariant transition operator $K$ on $X$. We need a $G$-invariant measure $d_{X}$ on $X$ which has a measurable decomposition $d_{X}=\int_{I} d_{X_{i}} d \lambda(i)$, where $\lambda$ is a Radon measure supported by the whole of $I$. We also need a decomposition (4.4) of $K$, and a measurable cross-section $\left\{\bar{x}_{i}: i \in I\right\}$. In typical specific cases, these objects are usually at hand. Then Theorem 5.3 and Proposition 5.5 remain valid without assuming compactness of $I$.

\section{The use of irreducibility and compactness conditions}

In this section, we start with an observation on irreducibility. Then we recall the Theorem of Jentzsch which extends the Perron Frobenius theorem to more general transition operators. This will be used to provide conditions that allow us to infer amenability from equality in (5.3.iii) and unimodularity from equality in (5.5.ii).

Let $\mu$ be any Radon measure on the metric space $X$. Then the transition operator $K$ on $X$ is called $\mu$-irreducible, ${ }^{4}$ if

$$
\mu(\{x \in X \backslash B: K(x, B)>0\})>0
$$

for every $B \in \mathcal{B}(X)$ with $\mu(B) \mu(X \backslash B)>0$.

\footnotetext{
${ }^{4}$ This definition, taken from Schaefer [36, p. 337], is slightly different from the one given in Nummelin [26].
} 
For the following, recall that $K^{0}=I d_{X}$, the identity operator on $X$, that is, $\operatorname{Id}_{X}(x, B)=\delta_{x}(B)$.

(6.1) Lemma. If $K$ is $\mu$-irreducible then for every $B \in \mathcal{B}(X)$ with $\mu(B)>0$ we have

$$
\mu\left(\left\{x \in X: \forall n \geq 0, K^{n}(x, B)=0\right\}\right)=0 .
$$

Proof. Write $X \backslash B^{\prime}$ for the above set whose measure is claimed to be 0 . Then $B \subset B^{\prime}$, whence $\mu\left(B^{\prime}\right)>0$. Assuming $\mu\left(X \backslash B^{\prime}\right)>0$, the assumption of $\mu$-irreducibility yields existence of $x \in X \backslash B^{\prime}$ with $K\left(x, B^{\prime}\right)>0$. But then also $K^{n}(x, B)>0$ for some $n$, a contradiction.

Next, consider compact integral operators on the compact space $I$ equipped with the finite measure $\lambda$. For the moment, let $(b(i, j))_{i, j \in I}$ be an arbitrary non-negative measurable kernel (density) that defines a bounded transition operator $\mathfrak{B} h(i)=\int_{I} b(i, j) h(j) d \lambda(j)$ on $L^{p}(I, \lambda)$. For $1<p<\infty$, set

$$
|\mathfrak{B}|_{p}^{p}=\int_{I}\left(\int_{I} b(i, j)^{q} d \lambda(j)\right)^{p / q} d \lambda(i) .
$$

From Schaefer $\left[36\right.$, p. 283] we learn that if $|\mathfrak{B}|_{p}<\infty$, then $\mathfrak{B}$ is a compact operator on $L^{p}(I, \lambda)$, and its adjoint $\mathfrak{B}^{*}$ (whose kernel is of course $b^{*}(i, j)=$ $b(j, i))$ is compact on $L^{q}(I, \lambda)$.

(6.2) Theorem [Jentzsch]. If $\mathfrak{B}$ is $\lambda$-irreducible and compact (in particular, if $|\mathfrak{B}|_{p}<\infty$ or $\left.\left|\mathfrak{B}^{*}\right|_{q}<\infty\right)$ then the spectral radius $\rho=\rho_{p}(\mathfrak{B})>0$ is an eigenvalue of $\mathfrak{B}$ whose eigenspace is one-dimensional and spanned by a function $h_{\rho}$ that is $\lambda$-almost everywhere $>0$.

See e.g. Schaefer [36, Thm. V.6.5].

We remark that in the specific case when $b(\cdot, \cdot)$ is essentially bounded $(\lambda \times \lambda)$ then $\rho$ has to be independent of $p$ and $h_{\rho}$ is essentially bounded $(\lambda)$. If in addition $b(\cdot, \cdot)$ is continuous on the (compact) space $I \times I$ then $\mathfrak{B}$ maps $L^{p}(I, \lambda)$ to $\mathcal{C}(I)$ for each $p$, and is also a compact operator on $\mathcal{C}(I)$ with the max-norm. In particular, $h_{\rho}$ is continuous and strictly positive in this case.

The following is also well known; for safety's sake we include the proof.

(6.3) Proposition (Subinvariance Theorem). Under the assumptions of (6.2), let $f \in L^{p}(I, \lambda), f \geq 0$ and $\lambda(f>0)>0$. Suppose that there is $t>0$ such that $\mathfrak{B} f \leq t \cdot f$ almost everywhere $(\lambda)$. Then

(a) $f>0$ almost everywhere $(\lambda)$,

(b) $t \geq \rho$, and

(c) $t=\rho$ if and only if $\mathfrak{B} f=t \cdot f$ almost everywhere $(\lambda)$. 
Proof. (a) Let $A=\{f>0\}$. Assume that $\lambda(I \backslash A)>0$, and let $A^{\prime}=\{i \in$ $I \backslash A: \mathfrak{B}(i, A)>0, t \cdot f(i) \geq \mathfrak{B} f(i)\}$. By $\lambda$-irreducibility, $\lambda\left(A^{\prime}\right)>0$. For $i \in A^{\prime}$, we have

$$
0=t \cdot f(i) \geq \mathfrak{B} f(i) \geq \int_{A} b(i, j) f(j) d \lambda(j)>0,
$$

a contradiction.

(b) Since $\rho\left(\mathfrak{B}^{*}\right)=\rho(\mathfrak{B})$, we can apply the Theorem of Jentzsch to $\mathfrak{B}^{*}$ and find a corresponding eigenfunction $h_{\rho}^{*}$ that is $\lambda$-a.e. $>0$. We have

$$
\begin{aligned}
t \int_{I} h_{\rho}^{*}(i) f(i) d \lambda(i) & \geq \int_{I} h_{\rho}^{*}(i) \mathfrak{B} f(i) d \lambda(i) \\
& =\int_{I} f(j) \mathfrak{B}^{*} h_{\rho}^{*}(j) d \lambda(j)=\rho \int_{I} f(j) h_{\rho}^{*}(j) d \lambda(j) .
\end{aligned}
$$

Since the last integral is positive by (a), (b) follows.

(c) If $\mathfrak{B} f=t \cdot f$ a.e. $(\lambda)$ then we must have $t=\rho$ by the above. Conversely, suppose that $\mathfrak{B} f \leq \rho \cdot f$, and that the inequality is strict in a set of positive measure. Then the argument used to prove (b), with $\rho$ in the place of $t$, would imply $\rho>\rho$, a contradiction.

(6.4) Corollary. Let $\mathfrak{A}$ and $\mathfrak{B}$ be two transition operators on $I$ with densities $a(i, j)$ and $b(i, j)$, respectively, with respect to $\lambda$. Assume that both satisfy the assumptions of Theorem (6.2).

(i) If $a(i, j) \leq b(i, j)$ for $\lambda \times \lambda$-almost every $(i, j)$ then $\rho_{p}(\mathfrak{A}) \leq \rho_{p}(\mathfrak{B})$.

(ii) If $a(i, j) \leq b(i, j)$ for $\lambda \times \lambda$-almost every $(i, j)$ and $\rho_{p}(\mathfrak{A})=\rho_{p}(\mathfrak{B})$ then $a(i, j)=b(i, j)$ almost everywhere $(\lambda \times \lambda)$, i.e., $\mathfrak{A}=\mathfrak{B}$.

Proof. Let $\rho=\rho_{p}(\mathfrak{B})$ and $h_{\rho}=h_{\rho, \mathfrak{B}}$. Then $\mathfrak{A} h_{\rho} \leq \mathfrak{B} h_{\rho}=\rho \cdot h_{\rho}$. Thus, (i) follows from Proposition 6.3(b). By (c), if the two spectral radii coincide, then $\mathfrak{A} h_{\rho}=\rho \cdot h_{\rho}$ a.e. $(\lambda)$. Therefore

$$
\int_{I}(b(i, j)-a(i, j)) h_{\rho}(j) d \lambda(j)=0 \text { for } \lambda \text {-almost every } i .
$$

Since $h_{\rho}>0$ a.e., (ii) follows.

We point out the obvious fact that if in addition $a(\cdot, \cdot)$ and $b(\cdot, \cdot)$ are continuous, then in statement (ii) they must coincide everywhere.

We now return to our $G$-invariant transition operator on $X$ as in Sections 4 and 5 . Let $z>0$ be such that

$$
\bar{K}(x, \cdot)=\sum_{n \geq 1} z^{n} K^{n}(x, \cdot)
$$

is a Radon measure on $X$. Note that if $\sigma_{2}(K)<\infty$ then we may take any $z<1 / \sigma_{2}(K)$. 
For the following, recall that we always assume that the measure $\lambda$ is supported by the whole of $I$, and consequently $\operatorname{supp}\left(d_{X}\right)=X$.

(6.6) Lemma. (a) If $K$ has a decomposition (4.4) then so does $\bar{K}$.

(b) If $K$ is $d_{X}$-irreducible then there is a subset $I_{0}$ of $I$ such that

$$
\lambda\left(I \backslash I_{0}\right)=0 \quad \text { and, for all } x \in \bigcup_{I_{0}} X_{i}, \quad \operatorname{supp}(\bar{K}(x, \cdot))=X .
$$

Proof. (a) is obvious.

For (b), note that in (6.5) it might have been more natural to start summation with $n=0$, i.e., with $K^{0}$, the identity operator. However, the latter decomposes only when $I$ is discrete. Write $\widetilde{K}=I d_{X}+\bar{K}$. Apply Lemma 6.1 to all $B$ in a countable set of open balls that generate the topology of $X$. This yields $\operatorname{supp}(\widetilde{K}(x, \cdot))=X$ for all $x$ in a set $B \subset X$ of full $d_{X^{-}}$-measure. Now $G$-invariance implies that $B=\pi^{-1}\left(I_{0}\right)$, where $\lambda\left(I \backslash I_{0}\right)=0$.

In order to pass from $\widetilde{K}$ to $\bar{K}$, fix $x \in B$. If $x$ is not an isolated point of $X$, then clearly $\operatorname{supp}(\bar{K}(x, \cdot))=X$.

If $x$ is isolated, then its orbit, say $X_{i}$, is discrete, and $\int_{\{y\}} d_{X}>0$ for all $y \in X_{i}$. As $d_{X}$ is supported by all of $X$, both $x$ and $X \backslash\{x\}$ have positive measure, whence $K(x, X \backslash\{x\})>0$ by $d_{X}$-irreducibility of $K$. On the other hand, $\widetilde{K}(y,\{x\})>0$ for all $y \in B$. Since $\bar{K}=K \widetilde{K}$, it follows that $\bar{K}(x,\{x\})>0$.

By an abuse of terminology, we shall say that $K$ is $\lambda$-irreducible, if $\mathfrak{B}[K]$ has that property on $I$. This can also be formulated without use of a decomposition (4.4), but if such a decomposition is given, then together with $\mathfrak{B}[K]$, also $\mathfrak{B}_{*}[K]$ and all the $\mathfrak{A}_{p}[K]$ and $\Sigma_{p}[K], 1<p<\infty$, are $\lambda$-irreducible.

(6.7) Theorem. Suppose that the transition operator $K$ on $X$ is $G$ invariant, that $I=G \backslash X$ is compact, and that we have a measurable decomposition of $d_{X}$ on $X$ as in (4.2) and a decomposition of $K$ as in (4.4). If $K$ is $\lambda$-irreducible,

$$
\left|\mathfrak{A}_{p}[K]\right|_{p}<\infty \quad \text { and } \quad \rho_{p}(K)=\rho_{p}\left(\mathfrak{A}_{p}[K]\right),
$$

then $\left\langle S\left[\bar{K}_{i, j}\right] S\left[\bar{K}_{i, j}\right]^{-1}\right\rangle^{-}$is amenable for $\lambda \times \lambda$-almost every $(i, j)$.

Proof. In view of (5.3.i), we may take $\bar{K}$ with $z<\left|\mathfrak{A}_{p}[K]\right|_{p}^{-1}$. The transition operators $\Sigma_{p}[\bar{K}]$ and $\mathfrak{A}_{p}[\bar{K}]$ are both $\lambda$-irreducible and compact on $L^{p}(I, \lambda)$, since

$$
\left|\Sigma_{p}[\bar{K}]\right|_{p} \leq\left|\mathfrak{A}_{p}[\bar{K}]\right|_{p}<\infty
$$


Using spectral theory and Lemmas 4.6 and 2.22, we get

$$
\rho_{p}(\bar{K})=\sum_{n} z^{n} \rho_{p}(K)^{n}=\sum_{n} z^{n} \rho_{p}\left(\mathfrak{A}_{p}[K]\right)^{n}=\rho_{p}\left(\mathfrak{A}_{p}[\bar{K}]\right) .
$$

Therefore Corollary 6.4 implies that $\bar{\sigma}_{p}(i, j)=\bar{a}_{p}(i, j)$ with respect to $\bar{K}$ for $\lambda \times \lambda$-almost every $(i, j)$. For these $(i, j)$, Theorem 2.12 yields amenability of $\left\langle S\left[\bar{K}_{i, j}\right] S\left[\bar{K}_{i, j}\right]^{-1}\right\rangle^{-}$.

(6.8) Remarks. (a) Recall Lemma 2.16, which implies that the "transpose" (adjoint over $I$ ) of $\mathfrak{A}_{p}[K]$ is $\mathfrak{A}_{p}[K]^{*}=\mathfrak{A}_{q}\left[K^{*}\right]$. Thus, the assumption $\left|\mathfrak{A}_{p}[K]\right|_{p}<\infty$ in Theorem 6.7 can also be replaced with $\left|\mathfrak{A}_{q}\left[K^{*}\right]\right|_{q}<\infty$.

(b) Contrary to the discrete case of Saloff-Coste and Woess [34], here we have no straightforward general argument that allows us to conclude that also the larger group $\left\langle S\left[\bar{K}_{i, j}\right]\right\rangle^{-}$has to be amenable for $\lambda \times \lambda$-almost every $(i, j)$ under the assumptions of Theorem 6.7. Namely, when $I$ is non-discrete, we cannot replace $\bar{K}$ with $I d_{X}+\bar{K}$ in the proof, since $I d_{X}$ will not factorize over $I$.

(c) Anyway, in many cases, we will have that $\operatorname{supp}\left(\bar{K}_{i, j}\left(\bar{x}_{i}, \cdot\right)\right)=X_{j}$ and thus $S\left[\bar{K}_{i, j}\right]=G$ for $\lambda \times \lambda$-almost every $(i, j)$, see $\S \S 7-8$ below. For this reason we have avoided to introduce additional space-consuming technicalities for passing to larger subgroups than the ones considered above.

The reasonable cases of (c) include Brownian motion on co-compact manifolds as well as random walks on quasi-transitive graphs. In particular, we have already collected all ingredients for deducing the theorem of Brooks [5] regarding the spectral gap on covers of compact Riemannian manifolds. We defer this to the last section for the sake of the inner logic of the presentation of our results.

For norms in the place of spectral radii, our necessary condition is more restrictive; as in Saloff-Coste and Woess [34], we can only treat the case $p=2$.

(6.9) Corollary. Assume that $K K^{*}$ is $\lambda$-irreducible and that the HilbertSchmidt norm $\left|\mathfrak{A}_{2}[K]\right|_{2}$ is finite. If

$$
\sigma_{2}(K)=\sigma_{2}\left(\mathfrak{A}_{2}[K]\right)
$$

then for $\lambda \times \lambda$-almost every $(i, j)$, the subgroup $\left\langle S\left[\left(\overline{K K^{*}}\right)_{i, j}\right] S\left[\left(\overline{K K^{*}}\right)_{i, j}\right]^{-1}\right\rangle^{-}$ of $G$ is amenable.

Proof. By Lemmas 2.16 and 2.22, $\mathfrak{A}_{2}\left[K K^{*}\right]=\mathfrak{A}_{2}[K K] \mathfrak{A}_{2}[K K]^{*}$, which is $\lambda$-irreducible and has finite Hilbert-Schmidt norm $\left|\mathfrak{A}_{2}[K]\right|_{2}^{2}$. We have

$$
\rho_{2}\left(K K^{*}\right)=\sigma_{2}(X)^{2}=\sigma_{2}\left(\mathfrak{A}_{2}[K]\right)^{2}=\rho_{2}\left(\mathfrak{A}_{2}\left[K K^{*}\right]\right),
$$

so that we can apply Theorem 6.7 to $K K^{*}$. 
Let us next study which implications equality in (5.5.ii) yields for the modular function. Because of the product on the right hand side of (5.5.ii), we cannot use the same argument as above in order to show that equality for $K$ also yields equality for $\bar{K}$ in its place.

(6.10) Proposition. Suppose that the transition operator $K$ on $X$ is $G$ invariant, that $I=G \backslash X$ is compact, and that we have a measurable decomposition of $d_{X}$ on $X$ as in (4.2) and a decomposition of $K$ as in (4.4). If $K$ is $\lambda$-irreducible,

$$
\left.\left|\mathfrak{B}[K] \mathbf{l}_{p}\right| \mathfrak{B}_{*}[K]^{*}\right|_{p}<\infty \quad \text { and } \quad \rho_{p}\left(\mathfrak{A}_{p}[K]\right)=\rho_{p}(\mathfrak{B}[K])^{1 / q} \rho_{q}\left(\mathfrak{B}_{*}[K]\right)^{1 / p}
$$

then there are constants $\alpha_{i, j}, i, j \in I$, such that for $\lambda \times \lambda$-almost every $(i, j)$, the following holds: $\left|G_{y}\right|=\alpha_{i, j}$ on $S\left[K_{i, j}\right]$, or equivalently, $\Delta \equiv 1$ on the group $\left\langle S\left[K_{i, j}\right] S\left[K_{i, j}\right]^{-1}\right\rangle^{-}$.

Proof. Let $c_{p}(i, j)=b(i, j)^{1 / q} b_{*}(j, i)^{1 / p}$ and recall from Proposition 2.17 that $a_{p}(i, j) \leq c_{p}(i, j)$. Write $\mathfrak{C}_{p}[K]$ for the resulting transition operator on $L^{p}(I, \lambda)$. Irreducibility of $\mathfrak{B}[K]$ implies that of $\mathfrak{C}_{p}[K]$ and $\mathfrak{A}_{p}[K]$. An exercise based on a few applications of Hölder's inequality shows that

$$
\left|\mathfrak{C}_{p}[K]\right|_{p} \leq|\mathfrak{B}[K]|_{p}^{1 / q}\left|\mathfrak{B}_{*}[K]^{*}\right|_{p}^{1 / p} .
$$

Therefore $\mathfrak{C}_{p}[K]$ and $\mathfrak{A}_{p}[K]$ are compact on $L^{p}(I, \lambda)$.

Next, let $c_{p}^{(n)}(\cdot, \cdot)$ be the density of $\mathfrak{C}_{p}^{n}$. By induction and Hölder,

$$
\begin{aligned}
c_{p}^{(n)}(i, j) & =\int_{I} c_{p}^{(n-1)}\left(i, i^{\prime}\right) c_{p}\left(i^{\prime}, j\right) d \lambda\left(i^{\prime}\right) \\
& \leq \int_{I}\left(b^{(n-1)}\left(i, i^{\prime}\right) b\left(i^{\prime}, j\right)\right)^{1 / q}\left(b_{*}\left(j, i^{\prime}\right) b_{*}^{(n-1)}\left(i^{\prime}, i\right)\right)^{1 / q} d \lambda\left(i^{\prime}\right) \\
& \leq b^{(n)}(i, j)^{1 / q} b_{*}^{(n)}(j, i)^{1 / p} .
\end{aligned}
$$

If $h \in L_{+}^{p}(I, \lambda)$ then

$$
\begin{aligned}
\mathfrak{C}_{p}^{n} h(i) & =\int_{I}\left(b^{(n)}(i, j) h(j)\right)^{1 / q}\left(b_{*}^{(n)}(j, i) h(j)\right)^{1 / p} d \lambda(j) \\
& \leq\left(\mathfrak{B}^{n} h(i)\right)^{1 / q}\left(\mathfrak{B}_{*}^{n *} h(i)\right)^{1 / p} .
\end{aligned}
$$

Therefore

$$
\left\|\mathfrak{C}_{p}^{n} h\right\|_{p} \leq \sigma_{p}\left(\mathfrak{B}^{n}\right)^{1 / q} \sigma_{q}\left(\mathfrak{B}_{*}^{n}\right)^{1 / p}\|h\|_{p}
$$

by another application of Hölder's inequality, whence

$$
\sigma_{p}\left(\mathfrak{C}_{p}^{n}\right) \leq \sigma_{p}\left(\mathfrak{B}^{n}\right)^{1 / q} \sigma_{q}\left(\mathfrak{B}_{*}^{n}\right)^{1 / p}
$$

for every $n$. 
We conclude that

$$
\rho_{p}\left(\mathfrak{A}_{p}[K]\right) \leq \rho_{p}\left(\mathfrak{C}_{p}[K]\right) \leq \rho_{p}(\mathfrak{B}[K])^{1 / q} \rho_{q}\left(\mathfrak{B}_{*}[K]\right)^{1 / p} .
$$

Consequently, if $\rho_{p}\left(\mathfrak{A}_{p}[K]\right)=\rho_{p}(\mathfrak{B}[K])^{1 / q} \rho_{q}\left(\mathfrak{B}_{*}[K]\right)^{1 / p}$ then Corollary 6.4 implies that $a_{p}(i, j)=c_{p}(i, j)$ for $\lambda \times \lambda$-almost every $(i, j)$. Proposition 2.17 implies the proposed statement.

In the last proposition, the condition $\left.\left|\mathfrak{B}[K] \mathbf{I}_{p}\right| \mathfrak{B}_{*}[K]^{*}\right|_{p}<\infty$ may be replaced with $\left|\mathfrak{B}[K]^{*}\right|_{q}\left|\mathfrak{B}_{*}[K]\right|_{q}<\infty$. Further conditions that imply unimodularity of the subgroup $\left\langle S\left[K_{i, j}\right] S\left[K_{i, j}\right]^{-1}\right\rangle$ can be obtained by transcribing (ii) and (iii) of Proposition 2.17.

\section{Transition operators with densities}

In sections 7 and 8 we assume that $G$ acts properly by isometries on the co-compact, proper $G$-space $X$. Here, we also assume to have a continuous decomposition $d_{X}=\int_{I} d_{X_{i}} d \lambda(i)$ of the $G$-invariant measure on $X$, as constructed in $\S 4 \mathrm{~A}$. Furthermore, we assume that our transition operator $K$ on $X$ has a density $k(x, y)$ with respect to $d_{X}$, that is,

$$
K f(x)=\int_{X} k(x, y) f(y) d_{X} y,
$$

where $k(\cdot, \cdot)$ is measurable on $X \times X$. In this case, the decomposition (4.4) is immediate, namely, $K_{i, j}$ is the transition operator whose density $k_{i, j}$ is the restriction of $k$ to $X_{i} \times X_{j}$. Also, the adjoint of $K$ is the transition operator with density $k^{*}(x, y)=k(y, x)$, and the density of $K_{i, j}^{*}$ is the corresponding restriction of $k^{*}$, that is,

$$
K_{i, j}^{*} f(x)=\int_{X_{j}} f(y) k(y, x) d_{X_{j}} y, \quad x \in X_{i} .
$$

As in the preceding sections, we use a measurable, relatively compact section $\left\{\bar{x}_{i}: i \in I\right\}$ containing one point in each orbit. A transition operator $K$ on $X \times X$, not necessarily having a density, is said to have bounded range, if there is $r>0$ such that $\operatorname{supp}(K(x, \cdot)) \subset \bar{B}(x, r)=\{y \in X: d(y, x) \leq r\}$ for every $x \in X$. In order to cover also the case of unbounded range, we define

$$
\Theta(r)=\sup \left\{K_{i, j}\left(\bar{x}_{i}, X_{j} \backslash B\left(\bar{x}_{i}, r\right)\right): i, j \in I\right\} .
$$

We say that $K$ has bounded tails if $\Theta(r)<\infty$ for some $r>0$, and uniform decay if $\lim _{r \rightarrow \infty} \Theta(r)=0$. 
In practice, the effort of proving bounded tails is more or less the same as proving uniform decay. We next provide a sufficient condition on the space $X$ and the density $k(\cdot, \cdot)$ that implies uniform decay. Let us say that $X$ is $\delta$-geodesic $(\delta>0)$, if for all $x, y \in X$, the following holds.

$$
\begin{gathered}
\text { If } n \delta \leq d(x, y) \text {, where } n \in \mathbb{N} \text {, then there is } w \in X \\
\text { such that } d(x, w)=n \delta \text { and } d(x, w)+d(w, y)=d(x, y) .
\end{gathered}
$$

By rescaling the metric, one may of course turn this into the case when $\delta=1$. "Purely" geodesic metric spaces satisfy condition (7.2) for all $\delta>0$. The definition also works for graphs, considered as discrete metric spaces (on the vertex set).

(7.3) Proposition. If $X$ is $\delta$-geodesic and there are $C, \kappa>0$ such that

$$
k(x, y) \leq C \exp \left(-d(x, y)^{1+\kappa} / C\right)
$$

then both $K$ and $K^{*}$ have uniform decay.

Proof. As above, we choose a compact set $B \subset X$ that intersects every orbit, and let $D=\operatorname{diam}(B)$.

If $i \in I$, then we define the relative growth function of $X_{i}$ (independent of the choice of $x \in X_{i}$ ) as

$$
V_{i}(r)=\int_{B(x, r) \cap X_{i}} d_{X_{i}}
$$

The term "relative" refers to the fact that this is the growth with respect to the "outer" metric on $X_{i}$, i.e., the restriction of the metric on $X$. We claim that there is $C>0$ such that

$$
V_{i}(r) \leq C e^{C r} \quad \text { for all } i \in I \text { and all } r \geq 1 .
$$

Suppose that (7.4) holds. Note that $K_{i, j}\left(x, X_{j} \backslash B(x, r)\right)$ is the same for all $x \in X_{i}$. Now let $x \in X_{i} \cap B$ and $y \in X_{j} \cap B$. Then $B(y, r-D) \subset B(x, r) \subset$ $B(y, r+D)$. Set $A(y, r)=B(y, r+1) \backslash B(y, r)$. We get

$$
\begin{aligned}
K_{i, j}\left(x, X_{j} \backslash B(x, r)\right) & \leq K_{i, j}\left(x, X_{j} \backslash B(y, r-D)\right) \\
& =\sum_{n=0}^{\infty} K_{i, j}\left(x, X_{j} \cap A(y, n+r-D)\right) .
\end{aligned}
$$

For $w \in A(y, n+r-D)$, we have $d(x, w) \geq n+r-2 D$. Therefore the last sum is

$$
\begin{aligned}
& \leq \sum_{n=0}^{\infty} C \exp \left(-\frac{1}{C}(n+r-2 D)^{1+\kappa}\right)\left(V_{j}(n+r-D+1)-V_{j}(n+r-D)\right) \\
& \leq \sum_{n=0}^{\infty} C^{2} \exp \left(-\frac{1}{C}(n+r-2 D)^{1+\kappa}+C(n+r-D+1)\right),
\end{aligned}
$$

which tends to 0 as $r \rightarrow \infty$. 
Thus, we are left with proving (7.4). We assume without loss of generality that $\delta=1$. As above, let $x \in X_{i} \cap B$ and $y \in X_{j} \cap B$. If $g \in G$ is such that $g x \in B(x, r)$, then $g y \in B(x, r+D) \subset B(y, r+2 D)$. Therefore by Lemma 2.6

$$
\begin{aligned}
V_{i}(r) & =\frac{1}{\left|G_{x}\right|} \int_{G} \mathbf{1}_{B(x, r)}(g x) d_{G} g \leq \frac{1}{\left|G_{x}\right|} \int_{G} \mathbf{1}_{B(y, r+2 D)}(g y) d_{G} g \\
& =\frac{\left|G_{x}\right|}{\left|G_{y}\right|} V_{j}(r+2 D) \leq M V_{j}(r+2 D)
\end{aligned}
$$

where $M=\max \left\{\left|G_{x}\right| /\left|G_{y}\right|: x, y \in B\right\}$.

For $x \in X$ and $r>0$, let $V(x, r)=\int_{B(x, r)} d_{X}$, the growth function of $X$ at $x$. It depends only on the orbit of $x$, and we may suppose that $x \in B$. Choose $x_{i} \in X_{i} \cap B$ for each $i$. We have for all $i, j \in I$

$$
\int_{B(x, r) \cap X_{j}} d_{X_{j}} \geq \int_{B\left(x_{j}, r-D\right) \cap X_{j}} d_{X_{j}} \geq V_{i}(r-3 D) / M,
$$

whence

$$
V(x, r)=\int_{I} \int_{B(x, r) \cap X_{j}} d_{X_{j}} d \lambda(j) \geq \frac{\lambda(I)}{M} V_{i}(r-3 D) .
$$

Next, let

$$
N(x, r)=\min \left\{m: \exists y_{1}, \ldots, y_{m} \text { with } \bar{B}(x, r) \subset \bigcup_{k=1}^{m} B\left(y_{k}, 1\right)\right\} .
$$

Clearly, $N(g x, r)=N(x, r)$ for $g \in G$. Also note that $V(x, r) \leq V(y, r+D)$ and $N(x, r) \leq N(y, r+D)$ for all $x, y \in B$.

Fix $x$ and let $y_{1}, \ldots, y_{m}, m=N(x, r)$ be as in the above definition. Since $X$ is 1-geodesic, if $w \in \bar{B}(x, r+s)$ then there is $v \in \bar{B}(x, r)$ such that $w \in \bar{B}(v, s)$, and there is $y_{k}$ such that $w \in \bar{B}\left(y_{k}, s+1\right)$. Consequently,

$$
\bar{B}(x, r+s) \subset \bigcup_{k=1}^{m} \bar{B}\left(y_{k}, s+1\right) .
$$

Therefore

$$
N(x, r+s) \leq \sum_{k=1}^{N(x, r)} N\left(y_{k}, s+1\right) \leq N(x, r) N(x, s+D+1) .
$$

This implies that $N(x, n(D+1)) \leq N(x, D+1)^{n}$, whence $N(x, r) \leq C^{\prime} e^{C^{\prime} r}$ for all $r \geq 1$, where $C^{\prime}>0$. To conclude, we only need that

$$
V(x, r) \leq \sum_{k=1}^{N(x, r)} V\left(y_{k}, 1\right) \leq V(x, D+1) N(x, r) .
$$

Regarding uniform decay of $K^{*}$, the proof is exactly the same, replacing $k(x, y)$ with $k^{*}(x, y)=k(y, x)$. 
(7.6) Remark. In (7.4) above, we have shown that $X$ has at most exponential growth. If $X$ is $\delta$-geodesic and has subexponential growth, that is,

$$
\limsup _{r \rightarrow \infty} V(x, r)^{1 / r}=1
$$

then $G$ has to be both amenable and unimodular.

To see this, one first verifies that for $r$ sufficiently large, the set $V=\{g \in$ $G: d(g x, x) \leq r\}$ is a compact neighbourhood of the identity that generates $G$. Next, the comparability of $V(x, r)$ with $V_{i}(r)$ that we have obtained in the proof of Proposition 7.3 yields that the homogeneous $G$-space $X_{i}$ has subexponential growth. Now one can use the results of Guivarc'h [18, $\S \mathrm{I}]$.

Using bounded tails, we can provide compactness criteria for the "reduced" transition operators on $I$. A function (on $X, X \times X$, etc.) will be called locally bounded, if it is bounded on every compact set.

(7.7) Lemma. If $K$ and $K^{*}$ have bounded tails and if the density of $K$ is locally bounded, then $b(\cdot, \cdot)$ and $b_{*}(\cdot, \cdot)$ are bounded. In particular, $|\mathfrak{B}[K]|_{p}$, $\mid \mathfrak{B}_{*}[K]^{*} \mathrm{I}_{p}$ and $\mid \mathfrak{A}_{p}[K] \mathrm{I}_{p}$ are all finite.

Proof. Let $D=\sup \left\{d\left(\bar{x}_{i}, \bar{x}_{j}\right): i, j \in I\right\}<\infty$, and let $r$ be such that $\Theta(r)<$ $\infty$. There is $C$ is such that $k\left(\bar{x}_{i}, y\right)<C$ for all $i \in I$ and $y \in B\left(\bar{x}_{i}, r+D\right)$. Using (7.5) - which does not rely on (7.2) - we get

$$
\begin{aligned}
b(i, j) & \leq \Theta(r)+K_{i, j}\left(\bar{x}_{i}, X_{j} \cap B\left(\bar{x}_{i}, r\right)\right) \leq \Theta(r)+K_{i, j}\left(\bar{x}_{i}, X_{j} \cap B\left(\bar{x}_{j}, r+D\right)\right) \\
& \leq \Theta(r)+C V_{j}(r+D) \leq \Theta(r)+M C V_{i_{0}}(r+3 D),
\end{aligned}
$$

where $i_{0} \in I$ is fixed. The "tail function" associated with $K^{*}$ is

$$
\Theta^{*}(r)=\sup _{i, j \in I} \int_{X_{i} \backslash B\left(\bar{x}_{j}\right)} k\left(y, \bar{x}_{j}\right) d_{X_{i}} .
$$

Hence, precisely as above, $b_{*}(i, j) \leq \Theta^{*}(r)+M C V_{i_{0}}(r+3 D)$.

Finally recall the inequality $a_{p}(i, j) \leq b(i, j)^{1 / q} b_{*}(j, i)^{1 / p}$ from Proposition 2.17 , showing that also $a_{p}(\cdot, \cdot)$ is bounded.

Jentzsch's Theorem now implies the following.

(7.8) Corollary. If $K$ is $\lambda$-irreducible, has bounded tails and locally bounded density, and $\mathfrak{A}$ is any one among the operators $\Sigma_{p}[K], \mathfrak{A}_{p}[K], \mathfrak{B}[K]$ and $\mathfrak{B}_{*}[K]$, then $\mathfrak{A}$ is a compact, positive operator on each of the spaces $L^{p}(I, \lambda)(1<p<\infty)$. The spectral radius $\rho(\mathfrak{A})$ is independent of the one among the latter spaces upon which $\mathfrak{A}$ acts, the eigenspace has dimension one, and there is a bounded eigenfunction which is strictly positive. 
(7.9) Remarks. (a) If the assumptions of Lemma 7.7 are replaced by the stronger assumptions that $K$ has uniform decay and continuous density, then it is quite easy to show that $b(\cdot, \cdot)$ is continuous on $I \times I$.

(b) If $k(\cdot, \cdot)$ is continuous, the conditions of Proposition 7.3 yield continuity of $\sigma_{p}(\cdot, \cdot)$ and $a_{p}(\cdot, \cdot)$ as well as of $b(\cdot, \cdot)$ and $b_{*}(\cdot, \cdot)$. For proving this, one can use the fact that $\left|G_{y}\right| /\left|G_{x}\right| \leq e^{C d(x, y)}$ for suitable $C>0$, if (7.2) holds. To see this, assume without loss of generality that $X$ is 1-geodesic. If $d(x, y)>1$ then take $w \in X$ such that $d(y, w)=1=d(x, y)-d(x, w)$. Let $g$ be such that $g w \in B$, where $B \subset X$ is compact and intersects every orbit. Then

$$
\frac{\left|G_{y}\right|}{\left|G_{x}\right|}=\frac{\left|G_{w}\right|}{\left|G_{x}\right|} \frac{\left|G_{g y}\right|}{\left|G_{g w}\right|} \leq \frac{\left|G_{w}\right|}{\left|G_{x}\right|} \max \left\{\frac{\left|G_{y_{0}}\right|}{\left|G_{x_{0}}\right|}: x_{0} \in B, d\left(y_{0}, x_{0}\right) \leq 1\right\} .
$$

(c) If all these kernels are continuous, then each of the the operators $\mathfrak{A}$ in Corollary 7.8 will also act on $\mathcal{C}(I)$, and its eigenfunctions are continuous.

If the conditions of Lemma 7.7 are satisfied, we can apply Theorem 6.7. Collecting all facts that we have accumulated so far, we obtain the following.

(7.10) Theorem. Suppose that

(i) $X$ is proper, $G$ acts properly by isometries, and $I=G \backslash X$ is compact,

(ii) the transition operator $K$ is $G$-invariant, $\lambda$-irreducible, with density $k(\cdot, \cdot)$

(iii) the density is locally bounded and both $K$ and $K^{*}$ have bounded tails, and

(iv) $G$ is connected, or $k(\cdot, \cdot)>0$ on $X$, or $S\left[K_{i, j}\right]$ generates $G$ as a semigroup for all $i, j$ in a set of positive $\lambda \times \lambda$-measure.

Then, for every $p \in(1, \infty)$,

$$
\rho_{p}(K) \leq \rho_{p}\left(\mathfrak{A}_{p}[K]\right) \leq \rho_{p}(\mathfrak{B}[K])^{1 / q} \rho_{q}\left(\mathfrak{B}_{*}[K]\right)^{1 / p} .
$$

The first inequality is an equality if and only if $G$ is amenable, and the second inequality is an equality if and only if $G$ is unimodular. Furthermore,

$$
\sigma_{2}(K) \leq \sigma_{2}\left(\mathfrak{A}_{2}[K]\right),
$$

and if $K K^{*}$ is $d_{X}$-irreducible then equality holds if and only if $G$ is amenable. then

If, in addition, $K$ is symmetric, i.e., $k(x, y)=k(y, x)$, and stochastic,

$$
\rho_{p}(K)=1 \Longleftrightarrow G \text { is amenable and unimodular. }
$$

Proof. The two inequalities come from Theorem 5.3 and Proposition 5.5.

Regarding the "if and only if" in case of equalities, note the small gap between the necessary and the sufficient conditions of Theorem 5.3.iv, resp. Proposition 5.5.iii on one hand and Theorem 6.7, resp. Proposition 6.10 on the other hand. 
When $G$ is connected, the gap disappears because existence of the density implies that the groups $\left\langle S S^{-1}\right\rangle \subset\langle S\rangle$ appearing in Theorem 5.3, as well as $\lambda \times \lambda$-almost all among the groups $\left\langle S\left[\bar{K}_{i, j}\right] S\left[\bar{K}_{i, j}\right]^{-1}\right\rangle$ and $\left\langle S\left[K_{i, j}\right] S\left[K_{i, j}\right]^{-1}\right\rangle$ that appear in Theorem 6.7 and Proposition 6.10, respectively, are openclosed and non-empty and hence must coincide with the whole of $G$. (Recall once more that the product of two subsets of $G$ with positive Haar measure contains a non-empty open set.)

If $S\left[K_{i, j}\right]$ generates $G$ as a semigroup then $S\left[K_{i, j}\right] S\left[K_{i, j}\right]^{-1}$ contains an open set. Therefore, the normal subgroup $N\left(K_{i, j}\right)$ defined as in (3.1) is open-closed and must have finite index in $G$ by Woess [42]. This closes the gap, as in the proof of Theorem 3.2.

The same works under the last of the conditions stated in (iv).

When $k(\cdot, \cdot)>0$ on $X$ all the above supports coincide with $G$, and so do the respective groups generated by them.

Finally, when $K$ is stochastic, then so is $\mathfrak{B}[K]$, whence $\rho_{p}(\mathfrak{B}[K])=1$. In the symmetric case, the same holds for $\mathfrak{B}_{*}[K]$.

In the symmetric case, we have of course $\sigma_{2}\left(K, d_{X}\right)=\rho_{2}\left(K, d_{X}\right)$, so that we also get $\sigma_{2}\left(K, d_{X}\right)=1$ if and only if $G$ is amenable and unimodular.

We remark that further variants or generalizations of the conditions (i)-(iv) can be formulated. For example, the case when $X$ is discrete and $K$ is irreducible has been completely settled by Saloff-Coste and Woess [34].

\section{Applications and computations}

From Theorem 7.10, we can now deduce as an immediate corollary one of the main results of this paper, namely the extension to co-compact manifolds of Theorems II and IV announced in the Introduction.

(8.1) Theorem. Let $\mathcal{M}$ be a complete, co-compact Riemannian manifold and $\mathfrak{L}_{\mathcal{M}}$ its (positive definite) Laplace-Beltrami operator.

Then the bottom of the $L^{2}$-spectrum of $\mathfrak{L}_{\mathcal{M}}$ is 0 (or equivalently, the heat kernel on $\mathcal{M}$ decays sub-exponentially in time) if and only if some (equivalently, every) closed, co-compact group of isometries of $\mathcal{M}$ is both amenable and unimodular.

Proof. As in the proof of Corollary $3.5, d_{X} \equiv d_{\mathcal{M}}$ is the volume element of $\mathcal{M}$, and we know from $\S 4$, resp. the Appendix, that it has a continuous decomposition of the form (4.2). We work with the heat operator $K_{1}(x, d y)=k_{1}(x, y) d_{X} y$ at time $t=1$, which is stochastic with symmetric, continuous density. 
A well-known long range estimate, says that for every $\varepsilon>0$, there is $C=C_{\varepsilon}>0$ such that

$$
k_{t}(x, y) \leq C \exp \left(-d(x, y)^{2} /(C t)\right) \quad \text { for all } x, y \in \mathcal{M}, t \geq \varepsilon,
$$

see Varopoulos [41] or Saloff-Coste [32, Thm. 5.2.10].

Also, $\mathcal{M}$ is of course geodesic. Therefore both $K$ and $K^{*}$ decay uniformly by Proposition 7.3, and Theorem 7.10 applies.

Note that in the last theorem it is not necessary that $G$ be a connected Lie group; it may also be discrete (= zero-dimensional). Since discrete groups are unimodular, Brooks' theorem stated in the introduction follows immediately.

(8.3) Corollary (Brooks [5]). Let $\mathcal{M}$ be the universal cover of the compact Riemannian manifold $\mathcal{M}_{0}$. Then the bottom of the $L^{2}$-spectrum of $\mathfrak{L}_{\mathcal{M}}$ is 0 if and only if the fundamental group of $\mathcal{M}_{0}$ is amenable.

An analogous result is valid for the Laplacian on simplicial complexes. We briefly explain the setup, see the book by Eells and Fuglede [16].

(8.4.i) $X$ is a $d$-dimensional simplicial complex, and each $d$-dimensional simplex has an isometric embedding into $\mathbb{R}^{d}$ as a non-degenerate Euclidean simplex. Every $k$-dimensional simplex $(k<d)$ is contained in some $d$ dimensional simplex.

(8.4.ii) The dual graph of $X$ is connected, that is, the graph whose vertices are the $d$-dimensional simplices, and two of them are neighbours if they have a common face $(=(d-1)$-dimensional simplex $)$.

(8.4.iii) Bounded geometry: every vertex of $X$ is contained in at most $M<\infty$ different simplices (of dimension $\leq d$ ). Inradius and outradius of each $d$-dimensional simplex, viewed as embedded in $\mathbb{R}^{d}$, are bounded below, resp. above by a positive, resp. finite constant. (In our situation, this is a consequence of co-compactness.)

(8.4.iv) The interior of each $d$-dimensional simplex is equipped with the standard Lebesgue measure and differential structure of (its embedding into) $\mathbb{R}^{d}$. We extend this to a measure $d_{X}$ on the whole complex.

(8.4.v) There is a natural Laplacian $\mathfrak{L}$ and associated heat kernel on $X$. Let $\mathcal{C}_{00}^{\infty}(X)$ be the space of all compactly supported functions on $X$ which are infinitely differentiable in each $d$-dimensional simplex and such that along the $(d-1)$-dimensional interior of any face, the sum of the inward normal derivatives with respect to all $d$-dimensional simplices containing that face is 0 . On $\mathcal{C}_{00}^{\infty}(X)$, the Laplacian is well defined, and via spectral and Dirichlet form theory one can show that it extends to a self-adjoint operator. 
(8.4.vi) The heat kernel associated with $\mathfrak{L}$ is continuous and has a Gaussian long range estimate as in (8.2). The latter is proved along the same lines as in the Riemannian case, see e.g. Saloff-Coste [32] and Sturm [39].

In the specific case of 2-dimensional complexes with non-positive curvature, an analogous Brownian motion theory has been studied by Brin and Kifer [4].

Since $X$ is a geodesic, proper metric space, precisely the same proof as that of Theoren 8.1 yields the following.

(8.5) Theorem. Let $X$ be a co-compact d-dimensional simplicial complex with properties (8.4.i-iv). Then the bottom of the $L^{2}$-spectrum of the Laplacian on $X$ is 0 (or equivalently, the heat kernel on $\mathcal{M}$ decays sub-exponentially in time) if and only if some (equivalently, every) closed, co-compact group of isometries of $X$ is both amenable and unimodular.

In the last theorem, note that $X$ can also be viewed as a $k$-dimensional complex, i.e., one can consider the Laplacian $\mathfrak{L}_{k}$ on its $k$-skeleton, where $1 \leq k \leq d$. If the statement of Theorem 8.5 holds for $\mathfrak{L}_{k}$ for some $k$, then it holds for all $k \leq d$. Note the particular case $d=1$, where we have the Laplacian on a graph whose edges are considered as copies of some interval. In all these cases, Salvatori's Theorem III stated in the introduction implies that the bottom of the spectrum of the Laplacian is 0 if and only if the 1skeleton of $X$, viewed as a discrete graph, has isoperimetric constant 0 . We also remark here that spectral properties of the one-dimensional Laplacian on infinite graphs have been studied by Cattaneo [7]. Finally, we add that Theorem 8.5 has obvious extensions to more general co-compact Riemannian complexes of the type described by Eells and Fuglede [16].

We next give a result on more general spaces than manifolds or simplicial complexes. Given $r>0$, the averaging operator over $r$-balls, $K=K_{(r)}$, is given as in Example 3.6 by

$$
K f(x)=\frac{1}{V(x, r)} \int_{B(x, r)} f(x) d_{X} x,
$$

where as above $V(x, r)=\int_{B(x, r)} d_{X}$. Note that $K$ is in general not symmetric (unless $V(\cdot, r)$ is constant). Thus, we cannot make use of the last statement in Theorem 7.10. Nevertheless, we can prove the following.

(8.6) Theorem. Suppose that $X$ is $\delta$-geodesic, $G$ is a closed subgroup of Iso $(X)$ and that $I=G \backslash X$ is compact. Let $K=K_{(r)}$ be the averaging operator over $r$-balls, where $r>\delta$. Then, for every $p \in(1, \infty)$, we have $\rho_{p}(K) \leq 1$ and

$$
\rho_{p}(K)=1 \Longleftrightarrow G \text { is amenable and unimodular. }
$$

If $X$ is a purely geodesic space then this is true for any $r>0$. The same holds for closed balls instead of open ones. 
Proof. The operator is $G$-invariant. It has bounded range and consequently it has bounded tails. The function $\mathrm{m}(x)=V(x, r)$ is constant on each orbit, and the density of $K$ is $k(x, y)=1 / \mathrm{m}(x)$, if $d(y, x)<r$, and $k(x, y)=0$, otherwise. We claim that

$$
M^{-1} \leq \mathrm{m}(x) \leq M \quad \text { for all } x \in X, \text { where } 0<M<\infty .
$$

It is clear that $V(x, r) \leq V\left(\bar{x}_{i_{0}}, r+D\right)<\infty$ for all $x$, where $D=\operatorname{diam}(I)$ as above, and $i_{0} \in I$ is fixed. For the lower bound, let $B \subset X$ be compact such that $\bar{x}_{i} \in B$ for all $i \in I$. We can find $y_{1}, \ldots, y_{\ell} \in X$ such that the balls $B\left(y_{k}, r / 2\right)$ cover $B$. If $\bar{x}_{i} \in B\left(y_{k}, r / 2\right)$ then $V\left(\bar{x}_{i}, r\right) \geq V\left(y_{k}, r / 2\right)$. Thus, $\mathrm{m}(x) \geq \min \left\{V\left(y_{k}, r / 2\right): k=1, \ldots, \ell\right\}$ for every $x \in X$. The latter number is positive, since $d_{X}$ is supported by all of $X$.

Let $k^{(m)}(\cdot, \cdot)$ denote the density of $K^{m}$. It is clearly bounded above. Also, $k^{(m)}(x, y)>0$ implies that $d(y, x)<m r$, so that $K^{m}$ has bounded range.

We claim that $d(x, y)<(m-1) \delta+r$ implies $k^{(m)}(x, y)>0$.

This is true for $m=1$. Suppose it holds for $m$, and let $d(x, y)<m \delta+r$. If $d(x, y)<(m-1) \delta+r$ then by assumption, $k^{(m)}(x, w)>0$ for $w \in B(y, \varepsilon)$, where $0<\varepsilon<r$, and

$$
k^{(m+1)}(x, y) \geq \int_{B(y, \varepsilon)} k^{(m)}(x, w) k(w, y) d_{X} w>0 .
$$

If $d(x, y) \geq(m-1) \delta+r$, which is $>m \delta$, then we can find $v \in X$ such that $d(x, v)=m \delta$ and $d(v, y)=d(x, y)-m \delta<r$. Thus, by the induction hypothesis, $k^{(m)}(x, w)>0$ and $k(w, y)$ in $B(v, \varepsilon)$, where $0<\varepsilon<r$, and $k^{(m+1)}(x, y)>0$ as above.

We now fix $m$ such that $\bar{r}=(m-1) \delta+r>\delta+2 D$. Then $\rho_{p}(K)=1$ $\Longleftrightarrow \rho_{p}\left(K^{m}\right)=1$. Therefore we can work with $K^{m}$ in the place of $K$. It has bounded density and bounded range.

Writing $b^{(m)}(i, j), b_{*}^{(m)}(i, j), a_{p}^{(m)}(i, j)$ and $\sigma_{p}^{(m)}(i, j)$ for the densities of the reduced operators on $I$ associated with $K^{m}$ (we warn the reader that these are not the same as the densities of $\mathfrak{B}[K]^{m}$, etc. !), all these numbers are $>0$ on $I \times I$, so that the reduced operators are all $\lambda$-irreducible and compact.

We note immediately that not only $\rho_{p}\left(\mathfrak{B}\left[K^{m}\right]\right)=1$, since this operator is stochastic, but also $\rho_{p}\left(\mathfrak{B}_{*}\left[K^{m}\right]\right)=1$, even though the adjoint of $K^{m}$ is not necessarily stochastic: we have $\mathrm{m}(x) k^{(m)}(x, y)=\mathrm{m}(y) k^{(m)}(y, x)$, i.e., $K^{m}$ is reversible. Setting $h(i)=\mathrm{m}(x)$, where $x \in X_{i}$ (a bounded function), we find that $b_{*}^{(m)}(i, j)=h(i) b^{(m)}(i, j) / h(j)$. Consequently, $\mathfrak{B}_{*}\left[K^{m}\right] h=h$, and $\rho_{q}\left(\mathfrak{B}_{*}\left[K^{m}\right]\right)=1$ by the Theorem of Jentzsch. 
To conclude, we show that for a suitable choice of reference points $\bar{x}_{i}$, $i \in I$, and for all $i, j$ in a set of positive $\lambda \times \lambda$-measure, the set

$$
S\left[\left(K^{m}\right)_{i, j}\right] \supset\left\{g \in G: d\left(g \bar{x}_{j}, \bar{x}_{i}\right)<\bar{r}\right\}
$$

contains an open, symmetric, relatively compact neighbourhood of the identity in $G$ that generates $G$.

Again, fix $i \in I$ and $\bar{x}_{i} \in X_{i}$, let $\varepsilon>0$ and set $V=\left\{g \in G: d\left(g \bar{x}_{i}, \bar{x}_{i}\right)<r_{1}\right\}$, where $r_{1}=2 D+\delta+\varepsilon$. Let $\ell \in \mathbb{N}$ be such that $D<\ell \delta \leq D+\delta$, and set $r_{0}=\ell \delta-D$.

We claim that for each $n \in \mathbb{N}$, if $g \in G$ and $d\left(g \bar{x}_{i}, \bar{x}_{i}\right) \leq n r_{0}$ then $g \in V^{n}$. This is true when $n \delta<r_{1}$, in particular when $n \leq 2 \ell-1$. Suppose it holds for $n-1$, where $n \delta \geq r_{1}$. Since $X$ is $\delta$-geodesic, there is $y \in X$ such that

$$
d\left(g \bar{x}_{i}, \bar{x}_{i}\right)=d\left(g \bar{x}_{i}, y\right)+d\left(y, \bar{x}_{i}\right) \text { and } d\left(g \bar{x}_{i}, y\right)=\ell \delta .
$$

Now there must be $z \in X_{i}$ such that $d(y, z) \leq D$. We get

$$
d\left(\bar{x}_{i}, g^{-1} z\right)=d\left(g \bar{x}_{i}, z\right) \leq \ell \delta+D<r_{1},
$$

so that there is $h \in V$ such that $g^{-1} z=h^{-1} \bar{x}_{i}$. On the other hand,

$$
d\left(\bar{x}_{i}, g h^{-1} \bar{x}_{i}\right)=d\left(\bar{x}_{i}, z\right) \leq d\left(\bar{x}_{i}, y\right)+D=d\left(g \bar{x}_{i}, \bar{x}_{i}\right)-r_{0} \leq(n-1) r_{0} .
$$

By the induction hypothesis, $g h^{-1} \in V^{n-1}$, and $g \in V^{n}$.

In $I$ with the factor space metric, we now consider the open ball $U$ of radius $\varepsilon=(\bar{r}-\delta-2 D) / 3$ centred at $i$. We can choose the representatives of the $G$-orbits in $X$ such that $\bar{x}_{j} \in B\left(\bar{x}_{i}, \varepsilon\right)$ for every $j \in U$. If $j, j^{\prime} \in U$ and $g \in V$ then $d\left(g \bar{x}_{j^{\prime}}, \bar{x}_{j}\right)<d\left(g \bar{x}_{i}, \bar{x}_{i}\right)+2 \varepsilon<r$, which means that $S\left[\left(K^{m}\right)_{j, j^{\prime}}\right] \supset V$ generates $G$ as a semigroup for all $\left(j, j^{\prime}\right) \in U \times U$.

Our basic assumption that $\operatorname{supp}\left(d_{X}\right)=X$ implies $\operatorname{supp}(\lambda)=I$, whence $U \times U$ has positive $\lambda \times \lambda$-measure.

Thus, we can apply Theorem 7.10.

If $X$ is purely geodesic then the above proof works for every $\delta>0$.

The last theorem can be extended to more general "averaging operators". Before concluding, we exhibit an example where Theorem 5.3 allows computation of norms and spectral radii, when $G$ is amenable.

(8.7) Example. Let $\mathbb{T}=\mathbb{T}_{s}$ be the homogeneous tree with degree $s+1$ $(s \geq 2)$, considered as a one-dimensional complex where each edge is a copy of the unit interval with Lebesgue measure. We write $V \mathbb{T}$ for its vertex set.

As in Cartwright, Kaimanovich and Woess [6] and Saloff-Coste and Woess [34], we choose a reference end $\omega$ of $\mathbb{T}$ and draw $\mathbb{T}$ in horocyclic layers with respect to $\omega$. We also choose a reference vertex $o$, its horocycle is numbered $H_{0}$, and we write $\mathfrak{h}(x)=n$ if $x \in H_{n}, n \in \mathbb{Z}$. See Figure 1 . 


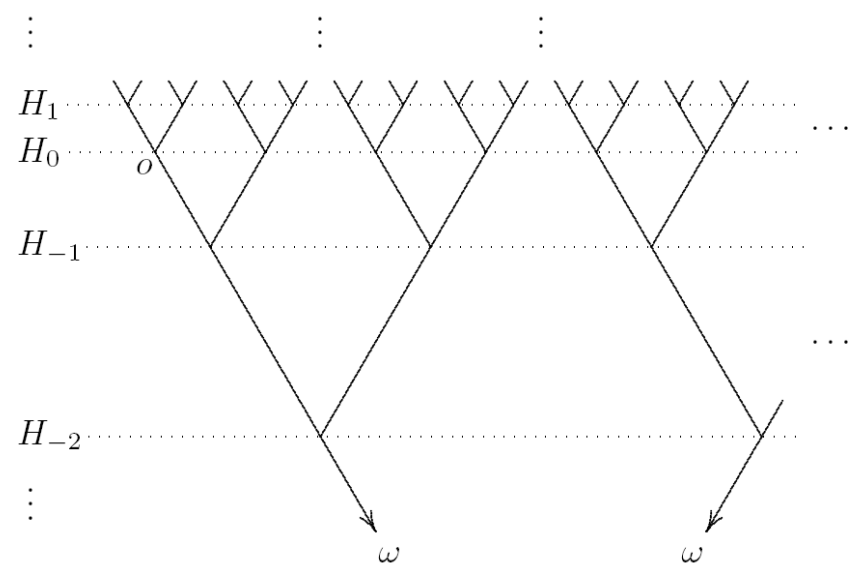

Figure 1

Thus, every vertex $v \in V \mathbb{T}$ has a unique predecessor $v-1 \in V \mathbb{T}$ with $\mathfrak{h}(v-1)=\mathfrak{h}(v)-1$, and is itself the predecessor of $\mathrm{s}$ vertices in $H_{\mathfrak{h}(v)+1}$. For every point $t \in \mathbb{T}$ and every $\tau \geq 0$, we define $t-\tau$ as the unique point on the geodesic ray from $t$ to $\omega$ at distance $\tau$ from $t$ in the obvious metric on $\mathbb{T}$. Thus, every $t \in \mathbb{T}$ can be written uniquely as $t=v-\alpha$, where $v \in V \mathbb{T}$ and $0 \leq \alpha<1$. If we define $\mathfrak{h}(t)=\mathfrak{h}(v)-\alpha$, then $\mathfrak{h}$ becomes a real valued function on $\mathbb{T}$ with $\mathfrak{h}(t-\tau)=\mathfrak{h}(t)-\tau$ for all $t \in \mathbb{T}$ and $\tau \geq 0$. The horocycles in $\mathbb{T}$ are the sets $H_{\kappa}=\{t \in \mathbb{T}: \mathfrak{h}(t)=\kappa\}$, where $\kappa \in \mathbb{R}$.

Consider the group $G=A f f(\mathbb{T})$ of all graph automorphisms (neighbourhood preserving permutations of the vertex set) $g$ which fix $\omega$. It acts transitively on $V \mathbb{T}$, is amenable and non-unimodular. By defining $g(v-\alpha)=g v-\alpha$, each $g \in G$ extends naturally to the whole of $\mathbb{T}$. The factor space $I=G \backslash \mathbb{T}$ is the circle with unit length, which we parametrize as $I=[0,1)$. The $G$-orbits are the sets

$$
T_{\alpha}=\bigcup_{n \in \mathbb{Z}} H_{n-\alpha}, \quad \alpha \in[0,1) .
$$

The obvious choice for the $G$-invariant measure $d_{\mathbb{T}}$ on $\mathbb{T}$ is the one whose restriction to each edge is a copy of Lebesgue measure on the unit interval, i.e., for a function $f: \mathbb{T} \rightarrow \mathbb{R}$,

$$
\int_{\mathbb{T}} f(t) d_{\mathbb{T}} t=\sum_{v \in V \mathbb{T}} \int_{0}^{1} f(v-\alpha) d \alpha .
$$

We now look for a decomposition of $d_{\mathbb{T}}$ according to (4.2). On $T_{0}=V \mathbb{T}$, we choose $d_{T_{0}}$ as the counting measure, and normalize left Haar measure on $G$ such that $\left|G_{o}\right|=1$. Then $\left|G_{v}\right|=\mathrm{s}^{-\mathfrak{h}(v)}$ for every $v \in V \mathbb{T}$, see e.g. Soardi and Woess [38]. Note that $G_{v-\alpha}=G_{v}$ for each $v \in V \mathbb{T}$ and $\alpha \in I$, but in order to obtain a continuous decomposition of $d_{\mathbb{T}}$, we should normalize such that $\left|G_{t}\right|=\mathrm{s}^{-\mathfrak{h}(t)}$ for all $t \in \mathbb{T}$. 
Thus, $d_{T_{\alpha}}$ is $\mathrm{s}^{-\alpha}$ times the counting measure on $T_{\alpha}$, and $d \lambda(\alpha)=\mathrm{s}^{\alpha} d \alpha$, where (as above) $d \alpha$ is Lebesgue measure on $I$. That is,

$$
\int_{\mathbb{T}} f(t) d_{\mathbb{T}} t=\int_{0}^{1} \underbrace{\underbrace{-\alpha}_{v \in V \mathbb{T}} f(v-\alpha)}_{\int_{T_{\alpha}} f(t) d_{T_{\alpha}} t} d \lambda(\alpha) .
$$

As representatives of the orbits we choose the points $\bar{t}_{\alpha}=o-\alpha, \alpha \in I$.

We now consider the averaging operator $K=K_{(1)}$ over balls of radius $r=1$. It is easy to compute the volume $V(t, 1)=\mathrm{s}+1$ of $B(t, 1)$ for all $t \in \mathbb{T}$. (However, when $r$ is not integer, then $V\left(\bar{t}_{\alpha}, r\right)$ depends on $\alpha$.) Thus, $K f(t)=\frac{1}{s+1} \int_{B(t, 1)} f d_{\mathbb{T}}$. Since $G$ is amenable, we can use Theorem 5.3 for computing $\rho_{p}(K), 1<p<\infty$. We calculate $a_{p}(\alpha, \beta)$ for $\alpha, \beta \in I$.

The general formula (for arbitrary radius $r$ in the place of $r=1$ ) is

$$
a_{p}(\alpha, \beta)=\frac{1}{V\left(\bar{t}_{\alpha}, r\right)} \sum_{n \in \mathbb{Z}} \mathrm{s}^{-\beta}\left|B\left(\bar{t}_{\alpha}, r\right) \cap H_{n-\beta}\right| \mathrm{s}^{-(n+\alpha-\beta) / p}
$$

since $\left|G_{t}\right| /\left|G_{\bar{t}_{\alpha}}\right|=1 / \mathrm{s}^{n+\alpha-\beta}$ for $t \in H_{n-\beta}$. If we conjugate the resulting operator on $I$ with respect to $\lambda$ by the (bounded) function $\alpha \mapsto \mathrm{s}^{\alpha / p}$ then the spectral radius remains the same. The last operator is the transition operator $\widetilde{\mathfrak{A}}_{p}$ on $I$ whose density with respect to Lebesgue measure (instead of $\lambda)$ is $\tilde{a}_{p}(\alpha, \beta)=\mathrm{s}^{\beta}\left(\mathrm{s}^{\alpha / p} a_{p}(\alpha, \beta) \mathrm{s}^{-\beta / p}\right)$. For $r=1$, we compute with a little combinatorial effort

$$
(1+\mathrm{s}) \tilde{a}_{p}(\alpha, \beta)= \begin{cases}\mathrm{s}^{1 / q}+\mathrm{s}, & 1-\beta \leq \alpha<\beta, \\ \mathrm{s}^{1 / q}+1, & \alpha<\beta<1-\alpha, \\ \mathrm{s}^{1 / p}+1, & \beta<\alpha<1-\beta, \\ \mathrm{s}^{1 / p}+\mathrm{s}, & 1-\alpha \leq \beta<\alpha .\end{cases}
$$

$\widetilde{\mathfrak{A}}_{p}$ is an integral operator with bounded density that is continuous on $[0,1) \times$ $[0,1)$ with exception of the diagonals $\beta=\alpha$ and $\beta=1-\alpha$. Thus, the "Perron-Frobenius" eigenfunction according to the Theorem of Jentzsch must be bounded. That is, we look for a positive, bounded function $h$ on $I$ such that

$$
\int_{0}^{1} \tilde{a}_{p}(\alpha, \beta) h(\beta) d \beta=\rho \cdot h(\alpha) \quad \forall \alpha
$$

and then we must have $\rho=\rho_{p}\left(\widetilde{\mathfrak{A}}_{p}\right)=\rho_{p}\left(\mathfrak{A}_{p}, \lambda\right)=\rho_{p}\left(K, d_{\mathbb{T}}\right)$. If we set $H(\alpha)=\int_{\alpha_{0}}^{\alpha} h(\beta) d \beta$, then our integral equation transforms into the following 
two equations: for $\alpha \leq 1 / 2$,

$$
\begin{aligned}
\rho h(\alpha) & =a(H(\alpha)-H(0))+b(H(1-\alpha)-H(\alpha))+c(H(1)-H(1-\alpha)) \\
\rho h(1-\alpha) & =a(H(\alpha)-H(0))+d(H(1-\alpha)-H(\alpha))+c(H(1)-H(1-\alpha)),
\end{aligned}
$$

where $a=\left(\mathrm{s}^{1 / p}+1\right) /(\mathrm{s}+1), b=\left(\mathrm{s}^{1 / q}+1\right) /(\mathrm{s}+1), c=\left(\mathrm{s}^{1 / q}+\mathrm{s}\right) /(\mathrm{s}+1)$ and $d=\left(\mathrm{s}^{1 / p}+\mathrm{s}\right) /(\mathrm{s}+1)$. In particular, $h$ must be continuous.

Setting $\Lambda=(\mathrm{s}+1) \rho, F(\alpha)=H(\alpha)$ and $G(\alpha)=H(1-\alpha)$ for $\alpha \in[0,1 / 2]$, we obtain a linear system of differential equations

$$
\begin{aligned}
& \Lambda F^{\prime}(\alpha)=\left(\mathrm{s}^{1 / p}+1\right) D+\left(\mathrm{s}^{1 / p}-\mathrm{s}^{1 / q}\right) F(\alpha)-(\mathrm{s}-1) G(\alpha), \\
& \Lambda G^{\prime}(\alpha)=-\left(\mathrm{s}^{1 / p}+1\right) D+(\mathrm{s}-1) F(\alpha)-\left(\mathrm{s}^{1 / p}-\mathrm{s}^{1 / q}\right) G(\alpha)
\end{aligned}
$$

subject to the boundary conditions $F(1 / 2)=G(1 / 2)$ and $s^{1 / q} G(0)-F(0)=D$. With $\varpi=\sqrt{\left(\mathrm{s}^{2 / p}-1\right)\left(\mathrm{s}^{2 / q}-1\right)} / \Lambda$, we find the real solutions

$$
\begin{aligned}
F(\alpha)=D /\left(s^{1 / q}-1\right) & +C(s-1) e^{i \varpi \alpha}+\bar{C}(s-1) e^{-i \varpi \alpha} \\
G(\alpha)=D /\left(s^{1 / q}-1\right) & +C\left(\left(s^{1 / p}-s^{1 / q}\right)-\mathfrak{i} \sqrt{\left(s^{2 / p}-1\right)\left(s^{2 / q}-1\right)}\right) e^{i \varpi \alpha} \\
& +\bar{C}\left(\left(s^{1 / p}-s^{1 / q}\right)+\mathfrak{i} \sqrt{\left(s^{2 / p}-1\right)\left(s^{2 / q}-1\right)}\right) e^{-i \varpi \alpha}
\end{aligned}
$$

where $C$ is arbitrary complex and $\bar{C}$ its conjugate. Inserting the two boundary conditions, we get $D=0$ and

$$
\varpi=\varpi_{n}=2 n \pi+\arctan \frac{\sqrt{\left(\mathrm{s}^{2 / p}-1\right)\left(\mathrm{s}^{2 / q}-1\right)}}{\mathrm{s}^{1 / p}+\mathrm{s}^{1 / q}}, \quad n \in \mathbb{Z} .
$$

Each $\varpi_{n}$ leads to a real solution, and among the corresponding eigenvalues $\rho$, the one when $n=0$ is largest. This is the value that we are looking for:

$$
\rho_{p}(K)=\frac{\sqrt{\left(\mathrm{s}^{2 / p}-1\right)\left(\mathrm{s}^{2 / q}-1\right)}}{\mathrm{s}+1} / \arctan \frac{\sqrt{\left(\mathrm{s}^{2 / p}-1\right)\left(\mathrm{s}^{2 / q}-1\right)}}{\mathrm{s}^{1 / p}+\mathrm{s}^{1 / q}} .
$$

In particular,

$$
\rho_{2}(K)=\sigma_{2}(K)=\frac{\mathrm{s}-1}{\mathrm{~s}+1} /(2 \arctan \sqrt{\mathrm{s}}-\pi / 2) .
$$

It may be useful to restate at this point the well-known analogous formulas for the transition operator $P$ of the simple random walk on the vertex set of $\mathbb{T}$,

$$
\rho_{p}(P)=\frac{\mathrm{s}^{1 / p}+\mathrm{s}^{1 / q}}{\mathrm{~s}+1}, \quad \text { in particular } \quad \rho_{2}(P)=\frac{2 \sqrt{\mathrm{s}}}{\mathrm{s}+1} .
$$

Similar computations can be undertaken when the radius of the ball is $r \neq 1$, but already for $r=1 / 2$ this leads to a linear system of 4 differential equations with non-constant coefficients. 
Outlook. In a forthcoming paper, we shall explain in detail a variety of further applications and examples. Here is a selection.

(a) Right convolution operators on a semi-simple Lie group NAK with finite centre are left-invariant under the action of the amenable group NA. Thus, the computation of norms and spectral radii can be reduced to those of (not necessarily K-invariant) transition operators on the compact factor space parametrized by K. The structure theory allows to extend such formulas and the resulting bounds to connected, locally compact groups. In this context, see also the recent paper by Chatterji, Pittet and Saloff-Coste [8].

(b) On graphs, seen as 1-dimensional complexes, we consider diffusions that behave like Brownian motion with drift on each edge (三 copy of the unit interval). If we have an amenable group of automorphisms that acts transitively, like in Example 8.7, then we can perform explicit computations in several cases, in particular, on homogeneous trees.

(c) Another interesting example combines Examples 3.6 and 8.7. This is the "hyperbolic - tree", or "treebolic" space appearing on page 421 in Farb and Mosher [17], on which the solvable Baumslag-Solitar groups act cocompactly, and generalizations thereof.

\section{Appendix: decomposition results}

In this appendix, we return to the situation of $\S 4$ and provide the details regarding the construction, resp. decomposition of invariant measures and transition operators that we have omitted there for the purpose of accelerating the access to the main results.

\section{A. Construction of invariant measures}

In order to construct an invariant measure on $X$, we choose a normalizing function $\bar{f} \in \mathcal{C}_{00}^{+}(X)$ such that $\operatorname{supp}\left(\bar{f}_{i}\right)$ is non-empty, so that $\bar{f}_{i}$ has positive, finite integral with respect to $d_{X_{i}}$ for every $i$. The latter measure is unique up to normalization, and we normalize it such that

$$
\int_{X_{i}} \bar{f}_{i}(x) d_{X_{i}} x=1 \quad \forall i \in I .
$$

A typical choice for $\bar{f}$ is a non-negative continuous function whose support is a ball $B(x, r)$ in $X$ with $r$ large enough such that $B(x, r) \cap X_{i}$ is non-empty for each $i$. Recall from Lemma 2.6 that with the above normalization,

$$
\left|G_{x}\right|=\int_{G} \bar{f}(g x) d_{G} g \quad \text { for every } x \in X .
$$


(9.3) Lemma. Let $f \in \mathcal{C}_{00}(X)$.

(1) The mapping $x \mapsto \int_{G} f(g x) d_{G} g$ is continuous on $X$.

(2) In particular, the function $x \mapsto\left|G_{x}\right|$ is continuous on $X$.

(3) The function $f^{b}(i)=\int_{X_{i}} f(x) d_{X_{i}} x$ is continuous on $I$.

Proof. Statement (1) is a straightforward exercise that uses properness of the action of $G$ on $X$. By (9.2), (2) arises as a special case of (1). Statement (3) also follows from Lemma 2.6, since $x \mapsto \frac{1}{\left|G_{x}\right|} \int_{G} f(g x) d_{G} g$ is continuous on $X$ and has constant value $f^{b}(i)$ on $X_{i}$.

Next, an invariant measure on $X$ is obtained by normalizing the measures on $X_{i}$ according to (9.1) and setting $d_{X}=\int_{I} d_{X_{i}} d \lambda(i)$ as in (4.2), where $\lambda$ is a Borel measure supported on the whole of $I$.

We now ask the following natural question: starting with a $G$-invariant measure $d_{X}$ on $X$ and a normalizing function $\bar{f}$ as in (9.1), is it always possible to decompose $d_{X}$ countinuously in as in (4.2) ? That is, we are now looking for a measure $\lambda$ on $I$ such that $d_{X}=\int_{I} d_{X_{i}} d \lambda(i)$.

Let $\pi: X \rightarrow I$ denote the natural projection. If $h \in \mathcal{C}(I)$ then (9.1) and (4.2) imply that we should have

$$
\int_{I} h(i) d \lambda(i)=\int_{X} h(\pi(x)) \bar{f}(x) d_{X} x .
$$

(9.5) Proposition. Suppose that $d_{X}$ is a $G$-invariant measure on $X$ and $\bar{f} \in \mathcal{C}_{00}^{+}(X)$ is a normalizing function. Then there is a unique measure $\lambda=\lambda^{\bar{f}}$ on $I$, defined by (9.4), such that $d_{X}=\int_{I} d_{X_{i}} d \lambda$, where each $d_{X_{i}}$ is normalized according to (9.1).

Proof. Given $\bar{f}$, we have the associated normalization of $d_{X_{i}}$ and measure $\lambda^{\bar{f}}$ on $I$. It follows from (9.4) that $\lambda^{c_{1} \bar{f}_{1}+c_{2} \bar{f}_{2}}=c_{1} \cdot \lambda^{\bar{f}_{1}}+c_{2} \cdot \lambda^{\bar{f}_{2}}$ for all $c_{1}, c_{2}>0$. Also, $\lambda^{\delta_{g} * \bar{f}}=\lambda^{\bar{f}}$ for every $g \in G$.

Set $U=\{\bar{f}>0\}$. By assumption, the family of sets $g U, g \in G$, form an open cover of $X$. By properness of $X$, there is a sequence $g_{n}, n \in \mathbb{N}$, in $G$ such that the sets $g_{n} U$ cover $X$, i.e., $\hat{f}=\sum_{n} 2^{-n} \delta_{g_{n}} * \bar{f}>0$ on all of $X$. Even though $\hat{f}$ is not a "true" normalizing function, since it does not have compact support, we can define $\lambda^{\hat{f}}$ analogously and find that $\lambda^{\bar{f}}=\lambda^{\hat{f}}$.

Now, we define a finite measure $\nu$ on $X$ by $\nu(B)=\int_{B} \hat{f}(x) d_{X} x$. Its projection onto $I$ is $\lambda^{\bar{f}}$, that is, $\lambda^{\bar{f}}(A)=\nu\left(\pi^{-1}(A)\right)$ for $A \in \mathcal{B}(I)$. Therefore, Dellacherie and Meyer [13, 70-72 on pp. 125-127] provide the existence of a family of probability measures $\nu_{i}$ on $X_{i}$, such that $i \mapsto \int_{X_{i}} f_{i} d \nu_{i}$ is measurable and

$$
\int_{X} f(x) d \nu(x)=\int_{I} \int_{X_{i}} f_{i}(x) d \nu_{i}(x) d \lambda^{\bar{f}}(i) \quad \text { for all } f \in \mathcal{C}(X) .
$$


We define a (Radon) measure $\mu_{i}$ on $X_{i}$ by $d \mu_{i}(x)=\left(1 / \hat{f}_{i}(x)\right) d \nu_{i}(x)$ and claim that it is $G$-invariant. Indeed, for all $g \in G, h \in \mathcal{C}(I)$ and $f \in \mathcal{C}_{00}(X)$, since $h(\pi(g x))=h(\pi(x))$,

$$
\begin{aligned}
\int_{I} h(i) & \int_{X_{i}} f_{i}(g x) d \mu_{i}(x) d \lambda^{\bar{f}}(i)=\int_{X} h(\pi(x)) f(g x) d_{X} x \\
=\int_{X} h(\pi(x)) f(x) d_{X} x & =\int_{I} h(i) \int_{X_{i}} f_{i}(x) d \mu_{i}(x) d \lambda^{\bar{f}}(i) .
\end{aligned}
$$

Therefore,

$$
\int_{X_{i}} f_{i}(g x) d \mu_{i}(x)=\int_{X_{i}} f_{i}(x) d \mu_{i}(x) \quad \text { for } \lambda^{\bar{f}} \text {-almost every } i \in I .
$$

A priori, the set of full $\lambda^{\bar{f}}$-measure where this last identity holds does depend on $f$ and $g$. But there is a standard procedure where one first starts with suitable dense and countable families of functions $f \in \mathcal{C}_{00}(X)$, resp. elements $g \in G$, and then extends to all functions and group elements by approximation. (This will be done in detail in a similar, but more complicated situation in the proof of Theorem 9.8 below.) Therefore, for $\lambda^{\bar{f}}$-almost every $i$, we have that $\mu_{i}=d_{X_{i}}$, and obviously $\int_{X_{i}} \bar{f}_{i} d_{X_{i}}=1$.

We add the following simple observation.

(9.6) Lemma. Given the $G$-invariant measure $d_{X}$ on $X$, let $\bar{f}, \bar{f}^{\prime} \in \mathcal{C}_{00}^{+}(X)$ be two normalizing functions. Then the measures $\lambda^{\bar{f}}$ and $\lambda^{\bar{f}^{\prime}}$, associated with $\bar{f}$ and $\bar{f}^{\prime}$ (respectively) according to (9.4), are mutually absolutely continuous.

Proof. Since the function $\hat{f}$ constructed in the above proof is continuous and $>0$ on $X$, there is $\varepsilon>0$ such that $\varepsilon \bar{f}^{\prime} \leq \hat{f}$. Therefore $\varepsilon \lambda^{\bar{f}^{\prime}} \leq \lambda^{\hat{f}}=\lambda^{\bar{f}}$.

Finally, the following lemma closes the circle and shows that our way of constructing, resp. decomposing invariant measures on $X$ is natural.

(9.7) Lemma. Suppose that we have a normalization of all $d_{X_{i}}, i \in I$, such that $x \mapsto\left|G_{x}\right|$ is continuous, and let $d_{X}=\int_{I} d_{X_{i}} d \lambda(i)$. Then there is a normalizing function $\bar{f}$ such that $\lambda=\lambda^{\bar{f}}$.

Proof. Let $\bar{f}_{0}$ be any normalizing function. By Lemma 9.3(1) and the assumed continuity of $x \mapsto\left|G_{x}\right|$, also the function

$$
\bar{h}(x)=\frac{1}{\left|G_{x}\right|} \int_{G} \bar{f}_{0}(g x) d_{G} g
$$

is continuous. Furthermore, it is constant on each orbit, i.e., $\bar{h}(g x)=\bar{h}(x)$ for every $g \in G$. Therefore, if we set $\bar{f}=\bar{f}_{0} / \bar{h}$, then this is a normalizing function, and applying Lemma 2.6(a) to an element of an orbit $X_{i}$, we find that (9.1) holds. 
Note, however, that the preceding results do not imply that any two $G$ invariant measures that are supported by the whole of $X$ must be mutually absolutely continuous. (In (4.2), take different measures $\lambda$ that are not mutually absolutely continuous.)

\section{B. Decomposition of transition operators}

Let us now consider the $G$-invariant transition operator $K$. We suppose to have $d_{X}=\int_{I} d_{X_{i}} d \lambda(i)$ as in (4.2), and we want to have a decomposition (4.4) of $K$. We give a necessary and sufficient existence condition in the case when $K$ is finite. For $x \in X$, consider the measure $\lambda_{x}$ on $I$ which is the image under $\pi$ of $K(x, \cdot)$. It is a finite measure by finiteness of $K$. If $A \in \mathcal{B}(I)$ then $\pi^{-1}(A)=\bigcup_{j \in A} X_{j}$ is invariant under every $g \in G$. From $G$ invariance of $K$ we see that $\lambda_{g x}=\lambda_{x}$, so that $\lambda_{x}$ depends only on $i=\pi(x)$, and we write $\lambda_{i}$ instead of $\lambda_{x}$.

(9.8) Theorem. Let $K$ be finite. For the existence of a decomposition of $K$ as in (4.4), it is necessary and sufficient that for $\lambda$-almost every $i \in I$, the measure $\lambda_{i}$ is absolutely continuous with respect to $\lambda$.

Proof. Necessity is easy: suppose that we have (4.4). Let $V \subset X$ be such that $\int_{X \backslash V} d_{X}=0$ and (4.4.iii) holds for every $x \in V$. For $i \in I$, let $V_{i}=V \cap X_{i}$. Then $\int_{X_{i} \backslash V_{i}} d_{X_{i}}=0$ on a set $I_{0} \subset I$ with $\lambda\left(I \backslash I_{0}\right)=0$. For $i \in I_{0}$, set $\varphi(i, j)=K_{j}\left(x, X_{j}\right)$, where $x \in X_{i}$. By (4.4.i), this is independent of the choice of $x \in X_{i}$, and by (4.4.ii), it is a measurable function on $I \times I$. Let $A \in \mathcal{B}(I)$, and choose $x \in V_{i}$. Then (4.4.iii) yields

$$
\lambda_{i}(A)=K\left(x, \pi^{-1}(A)\right)=\int_{A} K_{j}\left(x, X_{j}\right) d \lambda(j) .
$$

Therefore $\varphi(i, j)$ is the density of $\lambda_{i}$ with respect to $\lambda$.

Sufficiency needs more work. If we fix $x \in X$, then the theorem of Dellacherie and Meyer [13] used in Proposition 9.5 above guarantees the existence of a decomposition satisfying (4.4.iii) for this given $x$, but (i) and (ii) are not immediate from here. We have to extend carefully the method used by Dellacherie and Meyer.

To start with, let $\mathcal{H}$ be a countable, dense subset of the space $\mathcal{C}(I)$ of continuous functions on $I$ with the max-norm. Next, let $G^{0}$ be a countable, dense subgroup of $G$, and finally, let $\mathcal{C}^{0}$ be a countable subset of $\mathcal{C}_{00}(X)$ with the following properties: $(1)$ every $f \in \mathcal{C}_{00}(X)$ can be approximated in the sup-norm both from above and from below by functions in $\mathcal{C}^{0},(2) \mathcal{C}^{0}$ is a vector space over the rational numbers, (3) with any two functions, $\mathcal{C}^{0}$ also contains their point-wise maximum and minimum, (4) if $f \in \mathcal{C}^{0}$ and $g \in G^{0}$ then $\delta_{g} * f \in \mathcal{C}^{0},(5)$ if $f \in \mathcal{C}^{0}$ and $h \in \mathcal{H}$ then $f \cdot(h \circ \pi) \in \mathcal{C}^{0}$. 
First, let $f \in \mathcal{C}_{00}^{+}(X)$ be arbitrary, and consider the measures $\lambda_{x, f}$ on $I$ and $\Lambda_{f}$ on $X \times I$ defined by

$$
\lambda_{x, f}(A)=\int_{\pi^{-1}(A)} f(y) K(x, d y) \quad \text { and } \quad \Lambda_{f}(B \times A)=\int_{B} \lambda_{x, f}(A) d_{X} x,
$$

where $A \in \mathcal{B}(I), B \in \mathcal{B}(X)$.

Then $\lambda_{x, f}$ is absolutely continuous with respect to $\lambda$ for $d_{X}$-almost every $x$. Consequently $\Lambda_{f}$ is absolutely continuous with respect to $d_{X} \times \lambda$ and has a density $\varphi_{f}(x, j)$ that is measurable on $X \times I$. We extend $\Lambda_{f}$ and $\varphi_{f}$ to arbitrary functions in $\mathcal{C}_{00}(X)$ by setting $\Lambda_{f}=\Lambda_{f^{+}}-\Lambda_{f^{-}}$and $\varphi_{f}=\varphi_{f^{+}}-\varphi_{f^{-}}$. Note that $f \mapsto \Lambda_{f}$ is linear. Thus, if $f_{1}, f_{2} \in \mathcal{C}_{00}(X)$ and $a_{1}, a_{2} \in \mathbb{R}$, then $\varphi_{a_{1} f_{1}+a_{2} f_{2}}=a_{1} \varphi_{f_{1}}+a_{2} \varphi_{f_{2}}$ almost surely $\left(d_{X} \times \lambda\right)$. Furthermore, if $h \in \mathcal{C}(I)$ and $F \in \mathcal{C}_{00}(X \times I)$, then

$$
\begin{aligned}
\int_{X \times I} F(x, j) d \Lambda_{f \cdot(h \circ \pi)}(x, j) & =\int_{X} \int_{I} F(x, \pi(y)) h(\pi(y)) K(x, d y) d_{X} x \\
& =\int_{X \times I} F(x, j) h(j) d \Lambda_{f}(x, j),
\end{aligned}
$$

whence $\varphi_{f \cdot(h \circ \pi)}(x, j)=h(j) \varphi_{f}(x, j)$ for $d_{X} \times \lambda$-almost every $(x, j)$. Also, if $g \in G$ then $\lambda_{x, \delta_{g} * f}=\lambda_{g^{-1} x, f}$ since $\pi^{-1}(A)$ is $G$-invariant. Therefore

$$
\Lambda_{\delta_{g} * f}(B \times A)=\Lambda_{f}\left(g^{-1} B \times A\right),
$$

and $\varphi_{\delta_{g} * f}(x, j)=\varphi_{f}\left(g^{-1} x, j\right)$ for $d_{X} \times \lambda$-almost every $(x, j)$. We extend the action of $G$ to $X \times I$ by $g(x, j)=(g x, j)$.

Combining all these facts, we can find a Borel subset $U$ of $X \times I$ of full measure such that

$$
g U=U \text { for all } g \in G^{0},
$$

and, on the whole of $U$, for all $h \in \mathcal{H}, g \in G^{0}, f, f_{1}, f_{2} \in \mathcal{C}^{0}$ and $a_{1}, a_{2} \in \mathbb{Q}$,

$$
\begin{gathered}
\varphi_{\delta_{g} * f}=\delta_{g} * \varphi_{f} \\
\varphi_{f \cdot(h \circ \pi)}=h \varphi_{f} \\
\varphi_{a_{1} f_{1}+a_{2} f_{2}} a_{1}=\varphi_{f_{1}}+a_{2} \varphi_{f_{2}} \quad \text { and } \\
f \geq 0 \Longrightarrow \varphi_{f} \geq 0 .
\end{gathered}
$$

Fix $(x, j) \in U$. The map $f \mapsto \varphi_{f}(x, j)$ is $\mathbb{Q}$-linear and continuous with respect to uniform convergence on $\mathcal{C}^{0}$ by properties (1)-(3) of the latter space. Therefore it extends uniquely to a linear functional on $\mathcal{C}_{00}(X)$. For 
arbitrary $f \in \mathcal{C}_{00}(X)$, we write $\varphi_{f}(x, j)$ for this extension, when $(x, j) \in U$, and set $\varphi_{f}(x, j)=0$ when $(x, j) \in(X \times I) \backslash U$. This is justified, because it is a realization of the density of $\Lambda_{f}$ with respect to $d_{X} \times \lambda$. We remark that the extension is given by

$\varphi_{f}(x, j)=\sup \left\{\varphi_{f^{\prime}}(x, j): f^{\prime} \in \mathcal{C}^{0}, f^{\prime} \leq f\right\}=\inf \left\{\varphi_{f^{\prime \prime}}(x, j): f^{\prime \prime} \in \mathcal{C}^{0}, f^{\prime \prime} \geq f\right\}$,

and the supremum and infimum coincide by properties (1)-(3) of $\mathcal{C}^{0}$ (thus guaranteeing additivity of the extension). Thus, we obtain a non-negative functional $f \mapsto \varphi_{f}(x, j)$ on $\mathcal{C}_{00}(X)$ for every $(x, j) \in X \times I$, and for every $g \in G$, we have $\varphi_{\delta_{g} * f}=\delta_{g} * \varphi_{f}$. (A priori, by property (4) of $\mathcal{C}^{0}$ and by (9.9.b), the last identity holds on $G^{0}$. It extends to $G$ because $g_{n} \rightarrow g$ implies that $\delta_{g_{n}} * f \rightarrow \delta_{g} * f$ uniformly, when $f \in \mathcal{C}_{00}(X)$.) Finally, using denseness of $\mathcal{H}$ in $\mathcal{C}(I)$, we get that (4.4.c) holds for all $f \in \mathcal{C}_{00}(X)$ and $h \in \mathcal{C}(I)$.

Therefore, for every $(x, j) \in X \times I$, there is a Radon measure on $X$ that we denote by $K_{j}(x, \cdot)$, such that

$$
\varphi_{f}(x, j)=\int_{X} f(y) K_{j}(x, d y) \quad \text { for every } f \in \mathcal{C}_{00}(X) .
$$

This is the required transition operator $K_{j}$. By construction, $(x, j) \mapsto$ $K_{j} f(x)$ is measurable for every $f \in \mathcal{C}_{00}(X)$, and $K_{j}$ is $G$-invariant. Furthermore, since by construction,

$$
\int_{X} f(y) h(\pi(y)) K_{j}(x, d y)=h(j) \int_{X} f(y) K_{j}(x, d y)
$$

for $f \in \mathcal{C}_{00}(X)$ and $h \in \mathcal{C}(I)$, we have that the measure $K_{j}(x, d y)$ is supported by $X_{j}$ for every $x \in X$.

To conclude, let $B \in \mathcal{B}(X)$ and $f \in \mathcal{C}_{00}(X)$. Then we get

$$
\Lambda_{f}(B \times I)=\int_{B} \int_{I} \varphi_{f}(x, j) d \lambda(j) d_{X} x=\int_{B} \int_{I} K_{j} f_{j}(x) d \lambda(j) d_{X} x,
$$

where (recall) $f_{j}$ is the restriction of $f$ to $X_{j}$. On the other hand, $\Lambda_{f}(B \times I)=$ $\int_{B} K f(x) d_{X} x$. Since this holds for every $B \in \mathcal{B}(X)$, we get that

$$
K f(x)=\int_{I} K_{j} f_{j}(x) d \lambda(j) \quad \text { for } d_{X} \text {-almost every } x \in X .
$$

The "almost every" refers to a set $V_{f} \subset X$ whose complement has $d_{X^{-}}$ measure 0. Starting as above with the countable set $\mathcal{C}^{0}$ of functions and extending to all compactly supported functions by denseness, we find that $V_{f}$ may be replaced with a set of full measure that does not depend on $f$. 
Acknowledgements. Part of this work was done during a visit of Wolfgang Woess at Cornell University. He is grateful to Laurent Saloff-Coste and the Mathematics Department at Cornell for their warm hospitality. Another part was prepared during a stay of Wolfgang Woess at Institut Henri Poincaré - Centre Emile Borel, whose support is acknowledged. The second author acknowledges fruitful discussions on invariant measures with Peter Michor. Finally, we thank the referee(s) for useful comments.

\section{References}

[1] Berg, C. And Christensen, J. P. R.: On the relation between amenability of locally compact groups and the norms of convolution operators. Math. Ann. 208 (1974), 149-153.

[2] Berg, C. and Christensen, J. P. R.: Sur la norme des opérateurs de convolution. Invent. Math. 23 (1974), 173-178.

[3] Bourbaki, N.: Intégration. Hermann, Paris 1963.

[4] Brin, M. AND Kifer, Y.: Brownian motion, harmonic functions and hyperbolicity for Euclidean complexes. Math. Z. 237 (2001), 421-468.

[5] Brooks, R.: The fundamental group and the spectrum of the Laplacian. Comment. Math. Helv. 56 (1981), 581-598.

[6] Cartwright, D. I., Kaimanovich, V. A. and Woess, W.: Random walks on the affine group of local fields and of homogeneous tree. Ann. Inst. Fourier (Grenoble) 44 (1994), 1243-1288.

[7] Cattaneo, C.: The spectrum of the continuous Laplacian on a graph. Monatsh. Math. 124 (1997), 215-235.

[8] Chatterji, I. L., Pittet, Ch. and Saloff-Coste, L.: Connected Lie groups and property RD. To appear in Duke Math. J..

[9] Copson, E. T.: Metric Spaces. Cambridge Tracts in Mathematics and Mathematical Physics 57. Cambridge University Press, London, 1968.

[10] Cowling, M.: Michael Herz's "principe de majoration" and the KunzeStein phenomenon. In Harmonic analysis and number theory (Montreal, 1996), 73-88. CMS Conf. Proc. 21. Amer. Math. Soc., Providence, RI, 1997.

[11] Davies, E. B.: Non-Gaussian aspects of heat kernel behaviour. J. London Math. Soc. (2) 55 (1997), 105-125.

[12] DAY, M. M.: Convolutions, means and spectra. Illinois J. Math. 8 (1964), $100-111$.

[13] Dellacherie, C. And Meyer, P.-A.: Probabilités et Potentiel, Chapitres I à IV. Actualités Scientifiques et Industrielles 1372. Hermann, Paris, 1975.

[14] Derriennic, Y. and Guivarc'H, Y.: Théorème de renouvellement pour les groupes non moyennables. C. R. Acad. Sci. Paris, Sér. A-B 277 (1973), A613-A615. 
[15] Dieck, T. том: Transformation Groups. De Gruyter Studies in Mathematics 8. Walter de Gruyter, Berlin, 1987.

[16] Eells, J. And Fuglede, B.: Harmonic maps between Riemannian polyhedra. Cambridge Tracts in Mathematics 142. Cambridge University Press, Cambridge, 2001.

[17] Farb, B. And Mosher, L.: A rigidity theorem for the solvable BaumslagSolitar groups. Invent. Math. 131 (1998), 419-451.

[18] Guivarc'H, Y.: Croissance polynomiale et périodes des fonctions harmoniques. Bull. Soc. Math. France 101 (1973), 333-379.

[19] Guivarc'H, Y.: Sur la loi des grands nombres et le rayon spectral d'une marche aléatoire. In Conference on Random Walks (Kleebach, 1979), 47-98. Astérisque 74. Soc. Math. France, Paris, 1980.

[20] Herz, C.: Sur le phénomène de Kunze-Stein. C. R. Acad. Sci. Paris Sér. A-B 271 (1970), A491-A493.

[21] Herz, C.: The theory of $p$-spaces with an application to convolution operators. Trans. Amer. Math. Soc. 154 (1971), 69-82.

[22] Hewitt, E. And Ross, K. A.: Abstract Harmonic Analysis Vol. I: Structure of topological groups. Integration theory, group representations. Die Grundlehren der mathematischen Wissenschaften 115. Academic Press, New York; Springer-Verlag, Berlin-Göttingen-Heidelberg, 1963.

[23] Kesten, H.: Full Banach mean values on countable groups. Math. Scand. 7 (1959), 146-156.

[24] Kobayashi, T., Ono, K. and Sunada, T.: Periodic Schrödinger operators on a manifold. Forum Math. 1 (1989), 69-79.

[25] Lohoué, N.: Estimations $L^{p}$ des coefficients de représentation et opérateurs de convolution. Adv. in Math. 38 (1980), 178-221.

[26] Nummelin, E.: General irreducible Markov chains and nonnegative operators. Cambridge Tracts in Mathematics 83. Cambridge University Press, Cambridge, 1984.

[27] Parthasarathy, K. R.: Probability measures on metric spaces. Probability and Mathematical Statistics 3. Academic Press, New York, 1967.

[28] Pittet, Ch.: The isoperimetric profile of homogeneous Riemannian manifolds. J. Differential Geom. 54 (2000), 255-302.

[29] Raghunathan, M. S.: Discrete subgroups of Lie groups. Ergebnisse der Mathematik und ihrer Grenzgebiete 68. Springer-Verlag, New YorkHeidelberg, 1972.

[30] Reiter, H. and Stegeman, J. D.: Classical harmonic analysis and locally compact groups. London Mathematical Society Monographs New Series 22. The Clarendon Press, Oxford University Press, New York, 2000.

[31] Rudin, W.: Real and complex analysis. McGraw-Hill Book Co., New YorkToronto, Ont.-London 1966.

[32] Saloff-Coste, L.: Aspects of Sobolev-type inequalities. London Math. Soc. Lecture Note Series 289. Cambridge Univ. Press, Cambridge, 2002. 
[33] Saloff-Coste, L. And Woess, W.: Computing norms of group-invariant transition operators. Combin. Probab. Comput. 5 (1996), 161-178.

[34] Saloff-Coste, L. And Woess, W.: Transition operators, groups, norms, and spectral radii. Pacific J. Math. 180 (1997), 333-367.

[35] Salvatori, M.: On the norms of group-invariant transition operators on graphs. J. Theoret. Probab. 5 (1992), 563-576.

[36] Schaefer, H. H.: Banach Lattices and Positive Operators. Die Grundlehren der mathematischen Wissenschaften 215. Springer-Verlag, New York-Heidelberg, 1974.

[37] Seneta, E.: Non-negative matrices and Markov chains. Second edition. Springer Series in Statistics. Springer-Verlag, New York, 1981.

[38] Soardi, P. M. And Woess, W.: Amenability, unimodularity, and the spectral radius of random walks on infinite graphs. Math. Z. 205 (1990), 471-486.

[39] Sturm, K.-Th.: Analysis on local Dirichlet spaces. II. Upper Gaussian estimates for the fundamental solutions of parabolic equations. Osaka $J$. Math. 32 (1995), 275-312.

[40] Sy, P. W. and Sunada, T.: Discrete Schrödinger operators on a graph. Nagoya Math. J. 125 (1992), 141-150.

[41] Varopoulos, N. Th.: Small time Gaussian estimates of heat diffusion kernels. I: The semigroup technique. Bull. Sci. Math. 113 (1989), 253-277.

[42] Woess, W.: Périodicité de mesures de probabilité sur les groupes topologiques. In Random walks and stochastic processes on Lie groups (Nancy, 1981), 170-180. Inst. Élie Cartan 7. Univ. Nancy, Nancy, 1983.

[43] ŻUK, A.: A generalized Følner condition and the norms of random walk operators on groups. Enseig. Math. (2) 45 (1999), 321-348.

Recibido: 31 de marzo de 2004

Revisado: 10 de febrero de 2005

Laurent Saloff-Coste

Department of Mathematics

Cornell University

Ithaca, NY 14853, USA

lsc@math. cornell.edu

Wolfgang Woess

Institut für Mathematische Strukturtheorie

Technische Universität Graz

Steyrergasse 30, A-8010 Graz, Austria

woess@TUGraz . at

The research of L. Saloff-Coste is partially supported by NSF grant DMS 0102126. 